\title{
GOODS-ALMA: The slow downfall of star formation in $z=2-3$ massive galaxies
}

\author{
M. Franco ${ }^{1,2}$, D. Elbaz ${ }^{1}$, L. Zhou ${ }^{1,3,4}$, B. Magnelli ${ }^{5}$, C. Schreiber ${ }^{6}$, L. Ciesla ${ }^{1,7}$, M. Dickinson ${ }^{8}$, N. Nagar ${ }^{9,10,}$ \\ G. Magdis ${ }^{11,12,13,14}$, D. M. Alexander ${ }^{15}$, M. Béthermin ${ }^{7}$, R. Demarco ${ }^{9,10}$, E. Daddi ${ }^{1}$, T. Wang ${ }^{1,16}$, J. Mullaney ${ }^{17}$, \\ M. Sargent ${ }^{18}$, H. Inami ${ }^{19,20}$, X. Shu ${ }^{21}$, F. Bournaud ${ }^{1}$, R. Chary ${ }^{22}$, R. T. Coogan ${ }^{23}$, H. Ferguson ${ }^{24}$, S. L. Finkelstein ${ }^{25}$, \\ M. Giavalisco ${ }^{26}$, C. Gómez-Guijarro ${ }^{1}$, D. Iono ${ }^{27,28}$, S. Juneau ${ }^{1,8}$, G. Lagache ${ }^{7}$, L. Lin ${ }^{29}$, K. Motohara ${ }^{30}$, \\ K. Okumura ${ }^{1}$, M. Pannella ${ }^{31,32}$, C. Papovich ${ }^{33,34}$, A. Pope ${ }^{26}$, W. Rujopakarn ${ }^{35,36,37}$, J. Silverman ${ }^{37}$, and M. Xiao ${ }^{1,3}$ \\ (Affiliations can be found after the references)
}

Received 30 April 2020 / Accepted 31 August 2020

\begin{abstract}
We investigate the properties of a sample of 35 galaxies, detected with the Atacama Large Millimeter/Submillimeter Array (ALMA) at 1.1 mm in the GOODS-ALMA field ( area of $69 \mathrm{arcmin}^{2}$, resolution $=0.60^{\prime \prime}, \mathrm{rms} \simeq 0.18 \mathrm{mJy} \mathrm{beam}^{-1}$ ). Using the ultraviolet-to-radio deep multiwavelength coverage of the GOODS-South field, we fit the spectral energy distributions of these galaxies to derive their key physical properties. The galaxies detected by ALMA are among the most massive at $z=2-4\left(M_{\star, \text { med }}=8.5 \times 10^{10} M_{\odot}\right)$ and they are either starburst or located in the upper part of the galaxy star-forming main sequence. A significant portion of our galaxy population $(\sim 40 \%)$, located at $z \sim 2.5-3$, exhibits abnormally low gas fractions. The sizes of these galaxies, measured with ALMA, are compatible with the trend between the rest-frame $5000 \AA$ size and stellar mass observed for $z \sim 2$ elliptical galaxies, suggesting that they are building compact bulges. We show that there is a strong link between star formation surface density (at $1.1 \mathrm{~mm}$ ) and gas depletion time: The more compact a galaxy's star-forming region is, the shorter its lifetime will be (without gas replenishment). The identified compact sources associated with relatively short depletion timescales ( $100 \mathrm{Myr})$ are the ideal candidates to be the progenitors of compact elliptical galaxies at $z \sim 2$.
\end{abstract}

Key words. galaxies: high-redshift - galaxies: evolution - galaxies: star formation - galaxies: active - galaxies: fundamental parameters submillimeter: galaxies

\section{Introduction}

Over the last 8 billion years, the cosmic star formation density has decreased by a factor of $\sim 10$ (e.g., Madau \& Dickinson 2014). One of the major key questions in astrophysics is to understand why the Universe's star-forming activity reaches a peak around $z=2$ and why it is now so ineffective at generating stars.

Due to the lack of infrared (IR) surveys able to detect "typical" star-forming galaxies at $z>2$, the actual contribution of dust-obscured galaxies to the cosmic star formation history at these redshifts remains largely unknown, especially at high masses where galaxies are known to be metal-rich (e.g., Tremonti et al. 2004) and dust-rich (e.g., Boissier et al. 2004; Reddy et al. 2010). The star-formation rates (SFRs) of these high redshift galaxies are mostly estimated from ultraviolet (UV) measurements emitted by short-lived massive stars (e.g., Kennicutt \& Evans 2012). This UV emission is strongly affected by the presence of dust in the interstellar medium (ISM), which absorbs part of this emission that is to be re-emitted in IR. Therefore, to correctly estimate the SFR, a dust correction needs to be applied. This approach has proved effective for distant galaxies up to epochs close to reionization (e.g., Oesch et al. 2015; Bouwens et al. 2015; McLeod et al. 2015), but it suffers from caveats due to uncertainties on the attenuation law and the difficulties to constrain it (e.g., Cowie et al. 1996; Pannella et al. 2009). For this reason, constraining galaxy IR emission is essential to obtaining a robust star formation estimate.

The rest-frame peak of a galaxy's spectral energy distribution (SED) with a dust temperature between 30 and $50 \mathrm{~K}$ can vary between 72 and $125 \mu \mathrm{m}$ (e.g., Casey et al. 2014a), corresponding to an observed peak between $\sim 280$ and $500 \mu \mathrm{m}$ at $z=3$. To constrain the shape of the IR SED, at least one measurement must be carried out beyond this peak in the FIR part of the spectrum. This is why (sub)millimeter observations are necessary to constrain the IR luminosity of a galaxy. Thanks to the negative K-correction, submillimeter observations of galaxies are not affected by the flux decrease with increasing redshift over $2<z<10$ (Blain et al. 2002). With the Atacama Large Millimeter/Submillimeter Array (ALMA), it is now possible to detect galaxies with continuum emission below $1 \mathrm{mJy}$ and angular resolution lower than $1^{\prime \prime}$, which makes it possible to overcome the limit of confusion.

The study of distant and massive galaxies is essential to understanding our models of galaxy formation and evolution, as they are the ideal candidate progenitors of compact quiescent galaxies at $z \sim 2$ (Barro et al. 2013; Williams et al. 2014; van der Wel et al. 2014; Kocevski et al. 2017, see also Elbaz et al. 2018) and of present-day elliptical galaxies (Swinbank et al. 2006; Michałowski et al. 2010; Ricciardelli et al. 2010; Fu et al. 2013 ) that represent $60 \%$ of the total stellar mass in the local Universe (e.g., Fukugita et al. 1998; Hogg \& Turner 1998; Bell et al. 2003). In particular, one of the most critical questions about the growth of galaxies concerns the evolution of the gas fraction over cosmic time and of the efficiency of galaxies to transform this gas into stars (e.g., Somerville \& Davé 2015; Schinnerer et al. 2016; Tacconi et al. 2018). Therefore, the study of massive and distant galaxies is of the utmost importance to constrain galaxy evolution models. 
The Great Observatories Origins Deep Survey-South (GOODS-South) field benefits from deep and ultra-deep surveys over a large range of wavelengths (the Cosmic Assembly Near-infrared Deep Extragalactic Legacy Survey, CANDELS; Koekemoer et al. 2011; Grogin et al. 2011, PIs: S. Faber, H. Ferguson, the Spitzer Extended Deep Survey; Ashby et al. 2013, the GOODS-Herschel Survey; Elbaz et al. 2011, the Chandra Deep Field-South; Luo et al. 2017, ultra-deep radio imaging with the Karl G. Jansky Very Large Array; VLA; Rujopakarn et al. 2016 and the Hubble Ultra Deep Field, HUDF). This large effort allows us to study the whole SED of massive and distant galaxies by securing the cross-identification of ALMA detected galaxies thanks to its high angular resolution.

The GOODS-ALMA large survey covers $69 \mathrm{arcmin}^{2}$ in the deepest region of CANDELS, with a depth of $0.18 \mathrm{mJy}$, in which 20 sources were blindly detected (Franco et al. 2018, hereafter F18). A detailed description of this survey, detection techniques, first results, and the presentation of optically-dark galaxies revealed by ALMA are presented in F18. Going further in the analysis of these data, we used Spitzer/IRAC and VLA to extend our catalog to 16 additional sources detected down to $3.5 \sigma$ (see Franco et al. 2020, hereafter F20a).

Beyond cosmic noon $(z \gtrsim 2)$ most studies on the evolution of star formation density are based on Lyman break galaxy (LBG) samples (e.g., Steidel et al. 1999; Álvarez-Márquez et al. 2016; Bouwens et al. 2016). Already, evidence exists that above a stellar mass of typically $5 \times 10^{10} M_{\odot}$ the LBG technique misses the majority of massive dusty galaxies, because of their faintness and the redness of their UV slope (van Dokkum et al. 2006; Bian et al. 2013; Wang et al. 2016, 2019). Furthermore, recent studies with ALMA of a population of galaxies previously undetected by the Hubble Space Telescope (HST) has shed new light on our understanding of the origin and formation of massive galaxies (Wang et al. 2016, 2019; Fujimoto et al. 2016; Elbaz et al. 2018; Schreiber et al. 2018a; Williams et al. 2019). These optically dark galaxies constitute $20 \%$ of the sources detected in GOODS-ALMA (F18), 17\% if we include the sources detected down to $3.5 \sigma$ (see F20a). Despite the fact that they are undetected by the HST (at $5 \sigma$ limiting depth $H=28.2 \mathrm{AB}$ at $1.6 \mu \mathrm{m}$ ), they are detectable through their thermal dust emission thanks to the depth and capabilities of ALMA. The systematic study of massive galaxies $\left(M_{\star}>5 \times 10^{10} M_{\odot}\right)$ during this period of rapid transition between star-forming and quenched galaxies (Muzzin et al. 2013) is crucial to understand the mechanism by which star formation ceases in these galaxies.

Several surveys of the GOODS-South field have been carried on with ALMA around $1 \mathrm{~mm}$, resulting in a "wedding cake" distribution of surveys. A deep survey in the Hubble Ultra Deep Field (HUDF, 20 h, $4.5 \operatorname{arcmin}^{2}$, rms $=35 \mu \mathrm{Jy}, \lambda=1.3 \mathrm{~mm}$, Dunlop et al. 2017), a wider, shallower survey encompassing the HUDF, the ALMA $26 \operatorname{arcmin}^{2}$ survey of GOODS-S at one millimeter (ASAGAO, $45 \mathrm{~h}, 26 \operatorname{arcmin}^{2}, \mathrm{rms}=61 \mu \mathrm{Jy}, \lambda=1.2 \mathrm{~mm}$, Hatsukade et al. 2018) and finally the GOODS-ALMA survey itself encompassing both fields and covering the full area of GOODS-South with the deepest WFC $3 / H$-band coverage (PI: D. Elbaz, $20 \mathrm{~h}, 69 \operatorname{arcmin}^{2}, \mathrm{rms}=182 \mu \mathrm{Jy}, \lambda=1.1 \mathrm{~mm}, \mathrm{~F} 18$ ). In addition, two spectroscopic surveys (the ALMA Spectroscopic Surveys; ASPECS), a pilot (Walter et al. 2016) and large program (González-López et al. 2019), were performed with ALMA over an area of $\sim 1 \operatorname{arcmin}^{2}$ and $\sim 3 \operatorname{arcmin}^{2}$ respectively, inside the HUDF. This "wedding cake" approach allows us to both to collect information on extreme and rare galaxies in mapping large regions and also to have a precise view of more common and abundant galaxies with deep observations on a small area. Interestingly, extending the survey area allows the detection of more distant galaxies than deep observations of a smaller area. Indeed, while only $\sim 13 \%$ of the galaxies detected with ALMA have a redshift $\geq 3$ in the deep ALMA survey of HUDF (Dunlop et al. 2017), 40\% of the F18 galaxies are at $z \gtrsim 3$. In addition, ALMA surveys over large areas allow the detection of particularly massive and dusty galaxies that are rare in terms of surface density.

This paper is organized as follows: in Sect. 2, we describe the data used in this paper. In Sect. 3, we describe how we took advantage of our large multiwavelength coverage to fit the spectral energy distributions (SEDs) of the galaxies detected in GOODS-ALMA. In Sect. 4 we explain how we derived the main parameters of the galaxies ( $M_{\text {dust }}, M_{\text {gas }}$, SFR, depletion time). In Sect. 5, we discuss the results and interpret them as the evidence for a slow downfall of star formation in $z \sim 2-3$ massive galaxies.

Throughout this paper, we adopt a spatially flat $\Lambda \mathrm{CDM}$ cosmological model with $H_{0}=70 \mathrm{~km} \mathrm{~s}^{-1} \mathrm{Mpc}^{-1}, \Omega_{\mathrm{m}}=0.3$ and $\Omega_{\Lambda}=0.7$. We assume a Salpeter (Salpeter 1955) Initial Mass Function (IMF). We use the conversion factor of $M_{\star}$ (Salpeter $1955, \mathrm{IMF})=1.7 \times M_{\star}($ Chabrier 2003, IMF $)$ as suggested by Reddy (2006), Nordon et al. (2010), Caputi et al. (2015), Riechers et al. (2013), Seko et al. (2016), Elbaz et al. (2018). All magnitudes are quoted in the AB system (Oke \& Gunn 1983).

\section{Data}

\subsection{ALMA data}

This paper uses the ALMA observations obtained between August and September 2016 (Project ID: 2015.1.00543.S; PI: D. Elbaz), extending over an effective area of $69 \mathrm{arcmin}^{2}$, covering the deepest part of the CANDELS field - in the GOODS-South field - centered at $\alpha=3^{\mathrm{h}} 32^{\mathrm{m}} 30.0^{\mathrm{s}}, \delta=-27^{\circ} 48^{\prime} 00^{\prime \prime}(\mathrm{J} 2000)$. We perform this analysis in a $0.60^{\prime \prime}$-tapered mosaic reaching a $\mathrm{rms} \simeq 0.18 \mathrm{mJy} \mathrm{beam}^{-1}$. The complete description of this survey and the data reduction are presented in detail in F18, where the properties of 23 bright ALMA sources discovered as the result of the blind survey in this field are discussed and cataloged. Sources that were most likely false (indicated by an * in Table 2 in F18 and AGS22) are not taken into account in the rest of this paper. We consider these sources spurious because they have no counterparts at other wavelengths including mid-infrared. Among the 4 flagged sources, the 3 (AGS14, AGS16 and AGS19) which are in the $100 \mathrm{arcmin}^{2}$ of the SUPER-GOODS field of view have not been detected down to an $\mathrm{rms} \sim 0.56 \mathrm{mJy}$ at $850 \mu \mathrm{m}$ with SCUBA-2 (see the complete explanation of the rejection of these galaxies in F20a).

In addition, this catalog has been enriched with 16 galaxies, detected with a lower S/N, using the VLA and Spitzer/IRAC counterparts (see F20a for more details). In this work, we analyze a sample of 35 galaxies with redshifts between 0.6 and 4.7 $\left(z_{\text {med }}=2.7\right)$ and stellar masses ranging from $10^{10.3}$ to $10^{11.5} M_{\odot}$ $\left(M_{\star, \text { med }}=10^{10.93} M_{\odot}\right)$. The stellar mass and redshift distributions of these 35 galaxies are visible in Fig. 10 of F20a.

\subsection{Multiwavelength coverage}

We take advantage of the excellent multiwavelength coverage of the GOODS-South field to derive the physical properties of our galaxies. Below, we reproduce the list of the bands and filters used to observe this field (see Guo et al. 2013; Straatman et al. 2016), to fit the spectral energy distribution of these galaxies from 
the radio to the UV. We use the following bands: In UV, optical and near infrared (NIR), we used filters from VLT/VIMOS $(U$ and $R$; Nonino et al. 2009), from ESO/MPG/WFI $\left(U_{38}, V, R_{\mathrm{c}}\right.$; Baade et al. 1999; Hildebrandt et al. 2006), from HST/ACS $(F 435 W, F 606 W, F 775 W, F 814 W, F 850 L P$; Giavalisco et al. 2004; Wuyts et al. 2008; Guo et al. 2013), from HST/ WFC3 ( $F 098 M, F 105 W, F 125 W, F 160 W$; Grogin et al. 2011; Koekemoer et al. 2011), from Subaru Suprime-Cam (IA484, IA527, IA550, IA574, IA598, IA624, IA651, IA679, IA738, IA797, IA856; Cardamone et al. 2010), from CFHT/WIRCAM (K; Hsieh et al. 2012) from Magellan/FourStar (J1, J2, J3, Hs, Hl; Straatman et al. 2016), Spitzer/IRAC, channel 1 to 4 (Fazio et al. 2004), and Spitzer/MIPS (Rieke et al. 2004) filters. In far infrared (FIR), PACS (70 $\mu \mathrm{m}, 100 \mu \mathrm{m}, 160 \mu \mathrm{m}$; Poglitsch et al. 2010) and SPIRE ( $250 \mu \mathrm{m}, 350 \mu \mathrm{m}, 500 \mu \mathrm{m}$; Griffin et al. 2010). In radio, 5 and $10 \mathrm{~cm}$ images (Rujopakarn et al. 2016, and in prep.). In addition, where possible, we add the (sub)millimeter flux from previous pointings of the GOODS-South field (e.g., Zhou et al. 2020; Elbaz et al. 2018; Cowie et al. 2018; Barro et al. 2017; Talia et al. 2018) or during previous surveys (e.g., Hodge et al. 2013; Aravena et al. 2016; Dunlop et al. 2017; Hatsukade et al. 2018).

As the SPIRE beam is very large (18.1", 24.9", and 36.6" at $250 \mu \mathrm{m}, 350 \mu \mathrm{m}$, and $500 \mu \mathrm{m}$, respectively) and yielding a high confusion limit, we use the catalog of Wang et al. (in prep.), which is built with a state-of-the-art de-blending method. MIPS, radio and ALMA sources have been used to define prior positions to perform source extractions on the SPIRE images. Moving from shorter to longer wavelengths, predictions were made for the galaxy fluxes at longer wavelengths based on the redshift and photometry information at all available shorter wavelengths. The faint priors at longer wavelengths were then removed, which helps to break blending degeneracies and achieve deeper detection limits. Similar methods have been used to produce Herschel/SPIRE catalogs in GOODS-North (Liu et al. 2018).

\section{SED-fitting}

\subsection{Method}

We fit the spectral energy distributions using two different methods, depending on whether or not the galaxy has a Herschel counterpart. For galaxies that have a far-IR flux density measured by the Herschel space observatory, we employ the SEDfitting code CIGALE $^{1}$ (Code Investigating Galaxies Emission; Boquien et al. 2019). We use the stellar population models of Bruzual \& Charlot (2003) and the attenuation law of Calzetti et al. (2000). The IR SED fitting was performed using the dust infrared emission model given by Draine et al. (2014). We independently fit the wavelengths from the UV up to $16 \mu \mathrm{m}$, and from $24 \mu \mathrm{m}$ up to the millimeter wavelengths respectively (see Fig. 1 for an example and Fig. A.1 for the full sample). The radio portion has been added after the fitting process, using the FIR/radio correlation, with a constant ratio between FIR and radio luminosity of 2.34 (Yun et al. 2001). The parameters used in CIGALE were given by Ciesla et al. (2018) and are shown in Table 1.

In contrast, if the galaxy has no Herschel infrared counterpart, we fit the data with the dust spectral energy distribution library $^{2}$ presented in Schreiber et al. (2018b), and normalized to the ALMA flux density at $1.13 \mathrm{~mm}$ in the SED. We proceed iteratively. After fitting the galaxy with a star formation main sequence (MS; Noeske et al. 2007; Rodighiero et al. 2011;

$\begin{array}{ll}1 & \text { Publicly available at http://cigale.lam.fr } \\ 2 & \text { Publicly available at http://cschreib.github.io/s17-irlib/ }\end{array}$

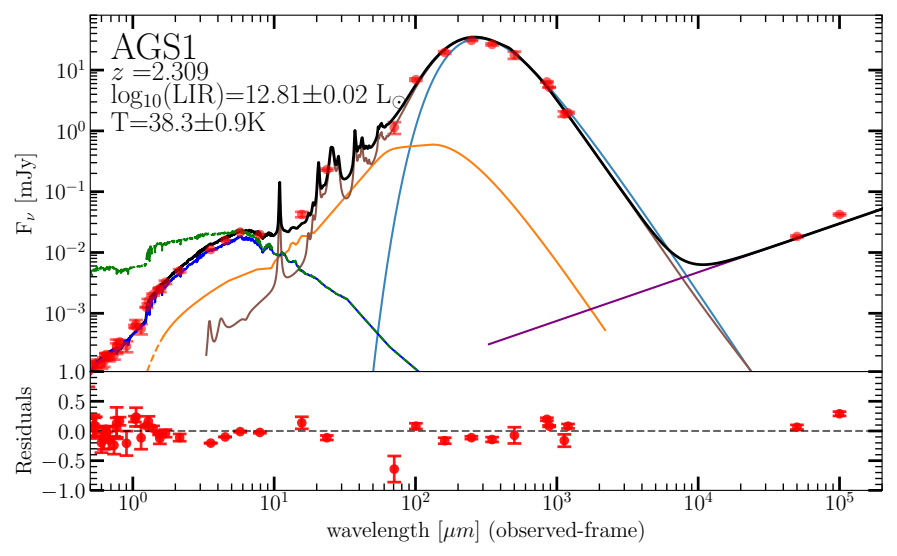

Fig. 1. SED of AGS1 shown as an example. The solid black line represents the best fit, which can be decomposed into the IR dust contribution (brown line), a stellar component uncorrected for dust attenuation (dark blue line), synchrotron emission (purple line) and the AGN contribution (orange line). An extrapolation of the AGN model of Kirkpatrick et al. (2015) to shorter wavelengths is displayed with an orange dashed line. In addition, we show the best fit of a modified black body, with $\beta=1.5$ (light blue line). The corrected UV emission is also shown in green. Bottom panel: residuals: (observation - model)/observation. The 34 other SEDs are given in the appendix (see Fig. A.1).

Table 1. Parameters used in the SED fitting procedures by CIGALE.

\begin{tabular}{ll}
\hline \hline Parameter & Value \\
\hline & Delayed SFH \\
Age $[\mathrm{Gyr}]$ & $100,250,500,1000,2000,3000$ \\
$\tau_{\text {main }}[\mathrm{Gyr}]$ & $100,500,1000,3000,5000,8000,10000$ \\
\hline & Dust attenuation \\
$E(B-V)_{*}$ & $0.01,0.1,0.3,0.4,0.6,0.7,0.9,1 ., 1.3$ \\
\hline & Dust emission \\
$U_{\text {min }}$ & $1,5,12,15,20,25,30,35,40,50$ \\
$\alpha$ & $1,1.5,2,2.5$ \\
$\gamma$ & $1 . \mathrm{e}-04,1 . \mathrm{e}-03,1 . \mathrm{e}-02,1 . \mathrm{e}-01,5 . \mathrm{e}-01,1$. \\
\hline
\end{tabular}

Elbaz et al. 2011) SED, we compute the distance to the main sequence $\left(R_{\mathrm{SB}}=\mathrm{SFR} / \mathrm{SFR}_{\mathrm{MS}}\right)$ using the output IR luminosity $(8-1000 \mu \mathrm{m})$ and the redshift. The $R_{\mathrm{SB}}$ and the redshift of the galaxy can be used to calculate the dust temperature ( $\left.T_{\text {dust }}\right)$ and IR8 $\left(L_{\mathrm{IR}} / L_{8}\right)$ from Eqs. (18) and (19) of Schreiber et al. (2018b). IR8 can be used as an indication of the compactness of distant galaxies (Elbaz et al. 2011). $T_{\text {dust }}$ and IR8 are therefore set to these newly calculated values in the SED-fitting process, and an updated SED is generated. The differences in the SED fitting derived quantities between the two methods are discussed in Sect. 4.1.

\subsection{AGN subtraction}

To fit an SED with an AGN component, we used the code decompIR by Mullaney et al. (2011). This code proposes to fit an AGN according to the spectrum of a sample of host galaxies representative of galaxies with an AGN. We consider that a galaxy hosts an AGN when one of the five decompIR models converges on a reasonable solution. The contribution of the AGN to the IR luminosity can lead to an overestimation of the dust infrared emission and therefore an overestimation of the SFR. The AGN SED used in decompIR does not include the wavelengths below 
$3 \mu \mathrm{m}$. To better characterize the contribution of AGN to the total infrared luminosity of galaxies, we need to know their behavior at rest-frame wavelengths lower than $3 \mu \mathrm{m}$, corresponding to the domain where the contribution of AGN is most important. Since this AGN model is only defined for wavelengths $>3 \mu \mathrm{m}$, we therefore used another AGN model for wavelengths shorter than $3 \mu \mathrm{m}$. We extrapolate AGN emission to shorter wavelengths, using an AGN model from Kirkpatrick et al. (2015), by fitting the flux of the AGN model from Kirkpatrick et al. (2015) to the one from decompIR at $5 \mu \mathrm{m}$ (the part least polluted by PAHs). The subtraction of the AGN contribution from the optical part of the galaxy spectrum remains highly uncertain, so we have chosen not to modify the stellar masses of galaxies hosting an AGN, whilst keeping in mind that they could be overestimated. The SED fitting is done in two iterations; during the first iteration, we remove the contribution of the AGN. We then apply the CIGALE code in order to derive the properties of the galaxy having eliminated the bias produced by the AGN.

\subsection{Dust temperature}

For the sake of simplicity and comparison with previous studies, we measure the dust temperature by fitting a modified black body (MBB) model, following:

$S_{v} \propto \frac{v^{3+\beta}}{\exp \left(\frac{h v}{k_{\mathrm{B}} T_{\text {dust }}}\right)-1}$,

where $k_{\mathrm{B}}$ is Boltzmann's constant, $h$ is the Planck's constant, $\beta$ is the dust emissivity spectral index, $T_{\text {dust }}$ is the dust temperature, and $S_{v}$ is the flux density. We have assumed a spectral index $\beta=1.5$ (e.g., Kovács et al. 2006; Gordon et al. 2010). We fit the flux densities at $\lambda_{\text {rest }} \geq 0.55 \lambda_{\text {peak }}$ using the MBB model as suggested by Hwang et al. (2010), and exclude the synchrotron contribution. The criteria we have defined to select the points to be modeled with a MBB are as follows: (i) at least one data point between $0.55 \times \lambda_{\text {peak }}$ and $\lambda_{\text {peak }}$ and (ii) at least one data point beyond $\lambda_{\text {peak }}$, with a wavelength lower than or equal to $3 \mathrm{~mm}$.

Galaxies selected in (sub)millimeter flux density are expected to be biased toward low dust temperatures (e.g., Chapman et al. 2003; Magdis et al. 2010; Casey et al. 2014b; McAlpine et al. 2019). Indeed, at fixed redshift and IR brightness, the (sub)millimeter flux of a galaxy with a colder dust temperature will be higher than that of a galaxy with a warmer dust temperature. We were interested to know whether the galaxies detected in this survey had unusual temperature characteristics, and if a possible temperature discrepancy could explain the properties of our sample. Indeed, the computation of both the dust mass and the gas mass (with the method we used), is temperature dependent.

We investigated where the galaxies detected in the GOODSALMA survey are located in the IR Luminosity-Temperature plane (Fig. 2, left panel) and in the Redshift-Temperature plane (Fig. 2, right panel). For comparison, we also plot the dust temperature of all the galaxies located in GOODS-ALMA with an MBB fit, as described above. We find that the galaxies detected by ALMA do not exhibit a systematic offset in the luminositytemperature and redshift-temperature plane compared to those undetected by ALMA. For an SMG, the dust temperature is correlated with the IR luminosity (e.g., Wardlow et al. 2011). We have found a median dust temperature of $40 \mathrm{~K}$ for our sample. However, we note that the spectral index $\beta$ has an influence on the temperature. We chose to fix it, at $\beta=1.5$ in order to have fewer free parameters in our fit and to compare all galaxies consistently. If we had taken $\beta=2$, on the other hand, the MBB temperatures would have been slightly lower (1-4 K lower). We note that we do not use this $T_{\text {dust }}$ temperature to determine dust masses (see Sect. 4.1).

\section{Derived parameters}

\subsection{Dust mass}

Following Draine et al. (2007), we adopt the maximum starlight intensity relative to the local interstellar radiation field $U_{\max }=10^{6} U_{\odot}$, and the power-law index $\alpha=2$ in Eq. (2). For the galaxies with a Herschel counterpart, the dust mass is estimated from the CIGALE fit (see Sect. 3) using the formula from Draine et al. (2007):

$$
\frac{\mathrm{d} M_{\mathrm{dust}}}{\mathrm{d} U}=(1-\gamma) M_{\mathrm{dust}} \delta\left(U-U_{\min }\right)+\gamma M_{\mathrm{dust}} \frac{\alpha-1}{U_{\min }^{1-\alpha}-U_{\max }^{1-\alpha}} U^{-\alpha},
$$

where $U_{\min } \leq U_{\max }, \alpha \neq 1$ is the exponent of the power law describing the intensity distribution of the interstellar radiation field, and $\gamma$ is the relative fraction of dust heated by each source. Draine et al. (2007) showed that $\alpha=2$ and $U_{\max }=10^{6}$ provided a good fit to a large sample of nearby galaxies from the Spitzer SINGS program (Kennicutt et al. 2003).

We compared the dust masses derived using CIGALE for galaxies with Herschel counterparts to those derived using the dust spectral energy distribution library presented in Schreiber et al. (2018b). We find a systematic offset of approximately 0.15 dex between the two methods, where dust masses derived using CIGALE are systematically larger. This may be caused by a slight underestimation of the ALMA fluxes (see F20a) directly affecting the dust measurement with the Schreiber et al. (2018b) dust library but compensated by the Herschel measurements when we use the full spectrum of the galaxy. In order to avoid biasing our study, and because we did not want to derive two physical quantities (the mass of dust and the mass of gas) from a single data point, we do not indicate the points without Herschel counterparts in the different figures and in the analysis. The differences however being small, we have presented the dust masses for galaxies with no Herschel counterpart in Table 2 as an indication.

\subsection{Gas mass}

As we discuss in Sect. 4.3.1, the ALMA detected galaxies are located in the SB region or in the upper part of the MS. To understand if their position is due to an increased star formation efficiency $\left(\mathrm{SFE} \equiv \mathrm{SFR} / M_{\mathrm{gas}}\right.$ ) or a large gas reservoir compared to normal MS galaxies, we computed their gas mass $M_{\text {gas }}$ as well as their gas fraction $f_{\text {gas }}$, defined by:

$f_{\mathrm{gas}}=\frac{M_{\mathrm{gas}}}{M_{\mathrm{gas}}+M_{\star}}$

To compute the gas mass, we assume a gas-to-dust ratio $\left(\delta_{\mathrm{GDR}}\right)$ depending only on metallicity. This method of derivation of the gas mass, in comparison with using the $\mathrm{CO}-$ to- $\mathrm{H}_{2}$ factor, which is observationally time consuming, can be used for a large survey. However, this method is based by a wide range of assumptions and limitations, such as the precise measurement of the dust mass, the assumption that the properties of the dust grains remain unchanged with redshift, that metallicity can be estimated at high redshift or that this relationship remains valid regardless of the distance to the main sequence (e.g., Magdis et al. 2011, 2012, 2017; Santini et al. 2014; Berta et al. 2016). 

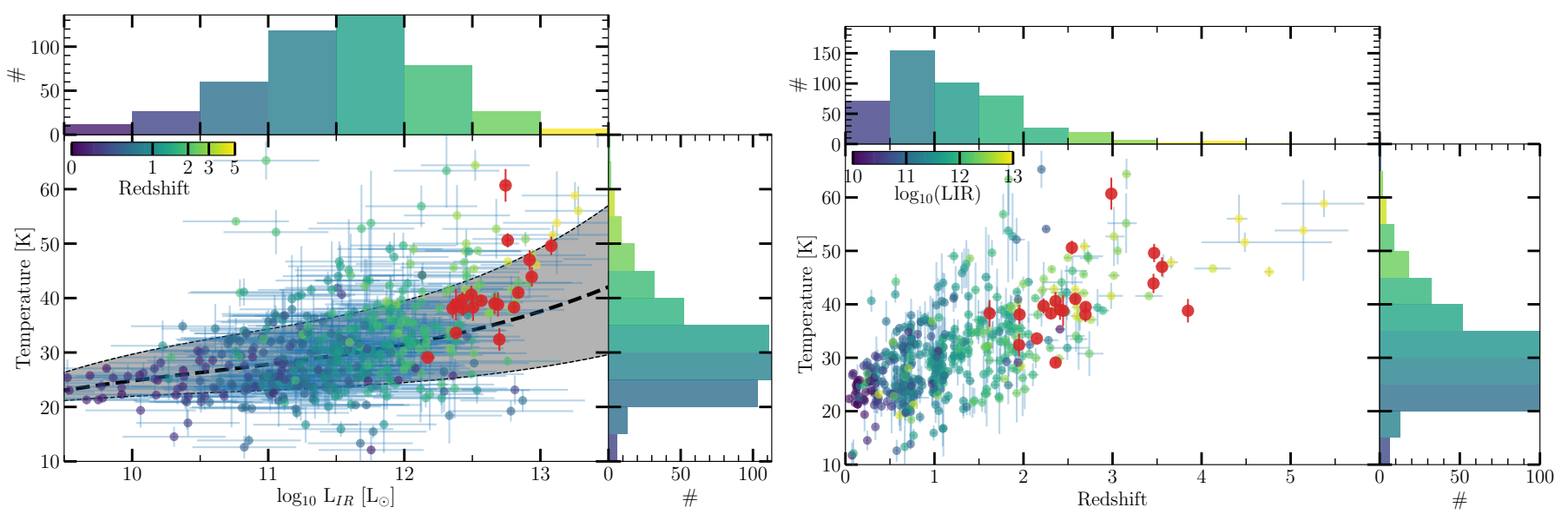

Fig. 2. Evolution of the dust temperature as a function of IR luminosity (left panel) and redshift (right panel) for the galaxies with a Herschel counterpart. The ALMA detections are shown in red. By comparison, we also plot the dust temperatures of all the galaxies within the GOODSALMA field, color-coded by redshift (left panel) or IR luminosity (right panel). We show the sliding median (and the $1 \sigma$ error) in black.

This ratio was directly derived by Leroy et al. (2011) in the local Universe, and can be applied to our sample, assuming that this relation is valid at all redshifts (e.g., Ivison et al. 2011; Casey et al. 2014b; Tan et al. 2014; Swinbank et al. 2014):

$\log _{10}\left(\delta_{\mathrm{GDR}}\right)=\frac{M_{\text {gas }}}{M_{\mathrm{dust}}}=(9.4 \pm 1.1)-(0.85 \pm 0.13)[12+\log (\mathrm{O} / \mathrm{H})]$,

where $M_{\text {gas }}=M\left(\mathrm{H}_{2}\right)+M\left(\mathrm{H}_{\mathrm{I}}\right)$. At the redshifts of this study, the atomic hydrogen can be considered negligible compared to the molecular form (e.g., Leroy et al. 2008; Obreschkow \& Rawlings 2009; Daddi et al. 2010).

We note that recent studies have found evidence for a steep increase in the gas-to-dust ratio of subsolar metallicity galaxies at $z \sim 2$ compared with this local relation (Coogan et al. 2019), but we do not expect this effect to be significant for our more massive, enriched galaxies. As we do not have direct metallicity measurements for our galaxies, we use the equation given by Genzel et al. (2012) to compute the metallicity:

$$
\begin{aligned}
12+\log (\mathrm{O} / \mathrm{H})= & -4.51+2.18 \log _{10}\left(M_{\star} / 1.7\right) \\
& -0.0896\left[\log _{10}\left(M_{\star} / 1.7\right)\right]^{2} .
\end{aligned}
$$

In this equation, we include a conversion factor (1/1.7) to transform the original formula from a Chabrier IMF to a Salpeter IMF. However, the metallicity can be underestimated for galaxies above the main sequence (e.g., Silverman et al. 2015), which could artificially increase the proportion of gas and conversely underestimate the gas depletion time (Elbaz et al. 2018). We compared our calculated metallicities to the metallicities obtained using the fundamental metallicity relation (FMR) of Mannucci et al. (2010):

$$
\begin{aligned}
12+\log _{10}(\mathrm{O} / \mathrm{H})= & 8.90+0.37 m-0.14 s-0.19 m^{2} \\
& +0.12 m s-0.054 s^{2}
\end{aligned}
$$

with $m=\log _{10}\left(M_{\star} / 1.7\right)-10$, and $s=\log _{10}(\mathrm{SFR} / 1.7)$. We applied an average correction factor of $-0.25 \pm 0.02$ to convert from the FMR derived using the Kewley \& Dopita (2002) metallicity calibration to the calibration of Pettini \& Pagel (2004), as given in Kewley \& Ellison (2008). The median metallicity ratio between these two methods is $1.03 \pm 0.01$, where the uncertainty corresponds to the standard deviation. For our galaxy sample, both methods are, therefore, in good agreement. However, the metallicities of these ALMA detected galaxies remain uncertain. Indeed, the metallicity evolution is poorly constrained for galaxies at high redshift, as well as for starburst galaxies and galaxies with AGN (e.g., Tan et al. 2013; Kewley et al. 2013). We keep in mind that the uncertainties on the determination of $M_{\text {gas }}$ are large, taking into account all of the assumptions used.

We also verified that the mass of gas derived by the method described above was in agreement with that derived using the method of Scoville et al. (2016). The Scoville et al. (2016) method is based on the assumption that continuum measurements of the Rayleigh-Jeans tail can be used to estimate the mass of dust and therefore, the mass of gas. Since this method is based on the Rayleigh-Jeans tail, it can only be used at long wavelengths $(\lambda>250 \mu \mathrm{m})$. However, if the dust emission is optically thin, the Scoville et al. (2016) method may underestimate the gas mass (Miettinen et al. 2017). At $1.13 \mathrm{~mm}$, the estimate of the gas mass can be written, according to Eqs. (6) and (16) of Scoville et al. (2016), as:

$M_{\mathrm{mol}}\left[M_{\odot}\right]=S_{v} \times 5.12 \times 10^{10} \times(1+z)^{-4.8} \times\left(d_{\mathrm{L}}\right)^{2} \frac{\Gamma_{\mathrm{RJ}}^{0}}{\Gamma_{\mathrm{RJ}}^{z}}$,

with $S_{v}$ the flux at $1.13 \mathrm{~mm}$ in mJy and $\Gamma_{\mathrm{RJ}}^{z}$ the correction for departure in the rest frame of the Planck function from RayleighJeans (Scoville et al. 2016):

$\Gamma_{\mathrm{RJ}}\left(T_{\mathrm{dust}}, v_{\mathrm{obs}}, z\right)=\frac{h v_{\mathrm{obs}}(1+z) /\left(k_{\mathrm{B}} T_{\mathrm{dust}}\right)}{e^{\left(h v_{\mathrm{obs}}(1+z) /\left(k_{\mathrm{B}} T_{\text {dust }}\right)\right)}-1}$

where $h$ is the Planck's constant and $k_{\mathrm{B}}$ is the Boltzmann constant. As explained in Scoville et al. (2016), the temperature we should use in this equation is not the temperature derived by the MBB fit for each individual galaxy but the mass-weighted temperature. We have therefore adopted a constant mass-weighted temperature value of $25 \mathrm{~K}$ (see Appendix A.2 in Scoville et al. 2016).

Using a fixed mass-weighted temperature of $25 \mathrm{~K}$, we find a difference between the calculated gas mass $\left(M_{\text {(gas, this work) }}\right)$ and that derived following Scoville et al. (2016): $M_{\text {(mol, Scoville) }} /$ $M_{\text {(gas,thiswork) }}=1.1 \pm 0.6$.

The gas mass is directly related to the depletion time $\left(\tau_{\text {dep }}\right)$ by:

$\tau_{\text {dep }}\left[\mathrm{yr}^{-1}\right]=\frac{M_{\text {gas }}}{\text { SFR }}$ 
Table 2. Derived properties of the GOODS-ALMA sources.

\begin{tabular}{|c|c|c|c|c|c|c|c|c|c|c|}
\hline ID & $z$ & $\begin{array}{c}\log _{10}\left(M_{\star}\right) \\
\left(M_{\odot}\right)\end{array}$ & $\begin{array}{c}\log _{10}\left(L_{\mathrm{IR}}\right) \\
\left(L_{\odot}\right)\end{array}$ & $\begin{array}{c}\text { SFR } \\
\left(M_{\odot} \mathrm{yr}^{-1}\right)\end{array}$ & $R_{\mathrm{SB}}$ & $\begin{array}{c}\log _{10}\left(M_{\text {dust }}\right) \\
\left(M_{\odot}\right)\end{array}$ & $\begin{array}{c}\log _{10}\left(M_{\mathrm{gas}}\right) \\
\left(M_{\odot}\right)\end{array}$ & $f_{\text {gas }}$ & $\begin{array}{l}T_{\text {dust }} \\
(\mathrm{K})\end{array}$ & $\begin{array}{c}S_{1.1 \mathrm{~mm}} \\
(\mathrm{mJy})\end{array}$ \\
\hline AGS1 & 2.309 & 11.15 & $12.81 \pm 0.02$ & $1103_{-60}^{+54}$ & & $9.2 \pm 0.1$ & $11.3 \pm 0.1$ & $.57_{-0.06}^{+0.07}$ & $38.3 \pm 0.9$ & $1.90 \pm 0.20$ \\
\hline AGS2 ${ }^{(\dagger)}$ & 2.918 & 10.68 & $12.98 \pm 0.14$ & & & & $11.0 \pm 0.1$ & & $44.8 \pm 4.6$ & $1.99 \pm 0.22$ \\
\hline AGS3 & 2.582 & 11.33 & $12.84 \pm 0.02$ & & & $9.1 \pm 0.1$ & $11.2 \pm 0.1$ & $0.42_{-0.05}^{+0.05}$ & $41.0 \pm 0.9$ & $1.84 \pm 0.21$ \\
\hline AGS4 & 3.556 & & $12.93 \pm 0.02$ & & & $8.9 \pm 0.1$ & $11.0 \pm 0.1$ & $0.44_{-0.04}^{+0.05}$ & $47.0 \pm 1.8$ & $1.72 \pm 0.20$ \\
\hline AGS5 & 3.46 & 11.13 & $12.94 \pm 0.02$ & & & $8.9 \pm 0.1$ & $11.0 \pm 0.1$ & $0.44_{-0.06}^{+0.06}$ & $43.9 \pm 1.8$ & $1.56 \pm$ \\
\hline AGS6 & 2.698 & 10.93 & $12.57 \pm 0.02$ & & & $9.0 \pm 0.1$ & $11.1 \pm 0.1$ & $0.59_{-0.08}^{+0.08}$ & $39.5 \pm$ & 1.27 \\
\hline AGS7 ${ }^{(\dagger)}$ & 3.29 & & 10 & & & & & & 37.4 & \\
\hline AGS8 & 1.95 & 11.53 & $12.70 \pm 0.02$ & & & $9.3 \pm 0.1$ & $11.3 \pm 0.1$ & $0.38_{-0.03}^{+0.03}$ & $32.4 \pm$ & $1.43=$ \\
\hline AGS9 & 3.847 & & $12.69 \pm 0$ & & & & $11.1 \pm 0.1$ & & $38.8 \pm$ & \\
\hline AGS10 & 2.41 & 11.25 & $12.66 \pm 0.02$ & & & $9.0 \pm 0.1$ & $11.0 \pm 0.1$ & $\begin{array}{l}+0.05 \\
-0.05\end{array}$ & $39.0 \pm$ & $0.88=$ \\
\hline AGS11 ${ }^{(\dagger)}$ & 3.472 & 10.24 & $12.94 \pm 0.07$ & & & $8.5 \pm$ & $10.8 \pm 0.1$ & & $50.1 \pm$ & 1.34 \\
\hline AGS12 & 2.543 & 10.77 & $12.76 \pm 0.02$ & $998_{-63}^{+40}$ & $8.1_{-0.5}^{+0.3}$ & $8.7 \pm 0.1$ & $10.9 \pm 0.1$ & $0.56_{-0.06}^{+0.07}$ & $50.6 \pm 1.3$ & $0.93=$ \\
\hline AGS13 & 2.225 & 11.40 & $12.43 \pm 0.02$ & & & $8.8 \pm 0.1$ & $10.8 \pm 0.1$ & $0.21_{-0.03}^{-0.00}$ & $39.7 \pm$ & 0.78 \\
\hline AGS15 (†) & 3.472 & 10.56 & $12.78 \pm 0.08$ & $\begin{array}{l}4_{110}^{180} \\
-179\end{array}$ & $9.5_{-1.7}^{+11.2}$ & $8.9 \pm 0.1$ & $11.1 \pm 0.1$ & $0.78_{-0.16}^{+0.19}$ & $38.9 \pm 2.4$ & $1.21^{(\star)} \pm 0.11$ \\
\hline AGS17 & 3.467 & 10.52 & $13.08 \pm 0.02$ & & $20.9_{-126}^{-3.1}$ & $9.0 \pm 0.1$ & $11.2 \pm 0.1$ & $0.84_{-0.09}^{-0.16}$ & $49.6 \pm 1.7$ & $2.30^{(\star)} \pm 0.44$ \\
\hline AGS18 & 2.696 & 11.11 & $12.43 \pm 0.03$ & $471_{-39}^{+19}$ & & $8.9 \pm 0.1$ & $11.0 \pm 0.1$ & $0.46_{-0.07}^{+0.08}$ & $38.1 \pm$ & $1.70^{(\star)} \pm 0.30$ \\
\hline AGS20 (†) & 2.73 & 10.76 & $12.59 \pm 0.08$ & & & $8.6 \pm 0.1$ & $10.8 \pm 0.1$ & $2_{-0.10}^{+0.11}$ & $40.6 \pm$ & $1.11 \pm 0.24$ \\
\hline AGS21 (†) & 3.689 & & & & & & & $\begin{array}{l}-0.06 \\
+0.06 \\
-0.06\end{array}$ & 43.7 & \\
\hline AGS23 & 2.36 & 11.26 & $12.17 \pm 0.05$ & & & $9.2 \pm 0.1$ & $11.2 \pm 0.1$ & & $29.1 \pm$ & \\
\hline & 3.472 & & & & & & & & & \\
\hline AGS25 (†) & 4.64 & & & & & $8.2 \pm 0.1$ & & & $51.5=$ & \\
\hline & 1.619 & & & & & & & & & \\
\hline AGS27 (†) & 4.73 & & $12.83 \pm 0.06$ & $1180_{-183}^{+144}$ & & & $10.6 \pm 0.1$ & & $48.0 \pm$ & \\
\hline AGS28 & 2.15 & & & & & & & & & \\
\hline AGS29 (†) & 1.117 & 10.77 & $11.96 \pm 0.10$ & & & $8.7 \pm 0.1$ & $10.9 \pm 0.1$ & $0.55_{-0.07}^{+0.08}$ & $31.5 \pm 2.4$ & $0.61 \pm$ \\
\hline AGS30 ${ }^{(\dagger)}$ & 0.646 & & & & & & & & & $0.67 \pm 0.17$ \\
\hline AGS31 & 2.45 & & $12.38 \pm 0.02$ & & & $8+01$ & $10.8 \pm 0.1$ & $0.21_{-0.03}^{+0.03}$ & $38.8 \pm 2.9$ & $0.72 \pm 0.19$ \\
\hline & 4.73 & & & & & & & $0.31_{-0.04}^{+0.04}$ & $44.9 \pm 2.5$ & \\
\hline AGS33 ${ }^{(\dagger)}$ & 2.68 & 10.71 & $12.40 \pm 0.05$ & & & $9.0 \pm 0.1$ & $11.1 \pm 0.1$ & $0.73_{-0.15}^{+0.17}$ & & $1.77 \pm 0.27$ \\
\hline AGS34 & 2.75 & & $12.15 \pm 0.04$ & & & $8.7 \pm 0.2$ & $10.9 \pm 0.2$ & & & $0.55 \pm 0.15$ \\
\hline AGS35 & 2.99 & 10.85 & $12.74 \pm 0.02$ & & & $8.3 \pm 0.1$ & $10.5 \pm 0.1$ & $0.29_{-0.03}^{+0.03}$ & $60.3 \pm 3.6$ & $1.16 \pm 0.21$ \\
\hline AGS36 ${ }^{(\dagger)}$ & 0.665 & 10.46 & $10.92 \pm 0.03$ & & $0.9_{-1}^{+}$ & $9.4 \pm 0.1$ & $11.7 \pm 0.1$ & & $\ldots$ & $0.74 \pm 0.21$ \\
\hline AGS37 & 1.956 & 11.19 & $12.36 \pm 0.02$ & & & $9.0 \pm 0.1$ & $11.0 \pm 0.1$ & & $38.0 \pm 1.6$ & $1.10 \pm 0.16$ \\
\hline AGS38 & 1.314 & & $11.64 \pm 0.02$ & & & $9.0 \pm 0.3$ & $11.1 \pm 0.3$ & & $\ldots$ & $1.00 \pm 0.16$ \\
\hline AGS39 & 2.36 & 10.57 & $12.49 \pm 0.02$ & $537_{-30}^{+24}$ & $7.4_{-0.4}^{+0.3}$ & $8.8 \pm 0.1$ & $11.0 \pm 0.1$ & $0.73_{-0.11}^{+0.13}$ & $40.6 \pm 1.7$ & $0.80 \pm 0.23$ \\
\hline
\end{tabular}

Notes. Columns: (1) source name; (2) redshifts (spectroscopic redshifts are shown to three decimal places); (3) stellar mass; (4) $L_{\mathrm{IR}}$ derived from SED fitting; (5) $\mathrm{SFR}=\mathrm{SFR}_{\mathrm{IR}}+\mathrm{SFR}_{\mathrm{UV}}$; (6) $R_{\mathrm{SB}}=\mathrm{SFR} / \mathrm{SFR} \mathrm{MS}$, where $\mathrm{SFR}_{\mathrm{MS}}$ is the average SFR of MS galaxies following Schreiber et al. (2015); (7) dust mass. For galaxies for which we used the dust spectral energy distribution library presented in Schreiber et al. (2018b) (labeled by a $\dagger$ in this table), the dust mass is multiplied by a factor of 2, to be consistent with the dust mass derived by the Draine et al. (2014) model (see Schreiber et al. 2018b for details); (8) gas mass derived from Eq. (4); (9) gas fraction defined by $f_{\mathrm{gas}}=M_{\mathrm{gas}} /\left(M_{\mathrm{gas}}+M_{\star}\right)$; (10) dust temperature derived from a MBB model assuming $\beta=1.5$. ${ }^{(\dagger)}$ Indicates galaxies without a Herschel counterpart and whose $L_{\mathrm{IR}}$ is determined only by the ALMA contribution. For these galaxies, we show the mass of gas as an indication but we do not use it in the rest of this paper; (11) flux density at $1.1 \mathrm{~mm}$. ${ }^{(\star)}$ Indicates changes in the flux density since F18. A summary of the fluxes (peak and integrated) as well as the sizes measured in the Main and Supplementary catalogs are given in Table A.1.

\subsection{SFR}

In this section, we detail the computation of the SFR of our sample. Although the IR part totally dominates the SFR, we took advantage of the UV coverage of GOODS-ALMA to calculate the total SFR $\left(\mathrm{SFR}_{\mathrm{tot}}=\mathrm{SFR}_{\mathrm{UV}}+\mathrm{SFR}_{\mathrm{IR}}\right)$.

\subsection{1. $S F R_{I R}$}

The infrared luminosity of each galaxy has been converted to SFR using the Kennicutt relation (Kennicutt 1998) below:

$\mathrm{SFR}_{\mathrm{IR}}\left[M_{\odot} \mathrm{yr}^{-1}\right]=1.72 \times 10^{-10} L_{\mathrm{IR}}$,

with $L_{\mathrm{IR}}$ in $L_{\odot}$, and

$L_{\mathrm{IR}}\left[L_{\odot}\right]=4 \pi d_{\mathrm{L}}^{2} \int_{8 \mu \mathrm{m}}^{1000 \mu \mathrm{m}} F_{v}(\lambda) \times \frac{c}{\lambda^{2}} \mathrm{~d} \lambda$,

where $d_{\mathrm{L}}$ is the luminosity distance.

In Fig. 3, we illustrate the distribution of SFRs as a function of redshift for the ALMA-detected galaxies. We also represent the theoretical detection limit of the galaxies present in the 


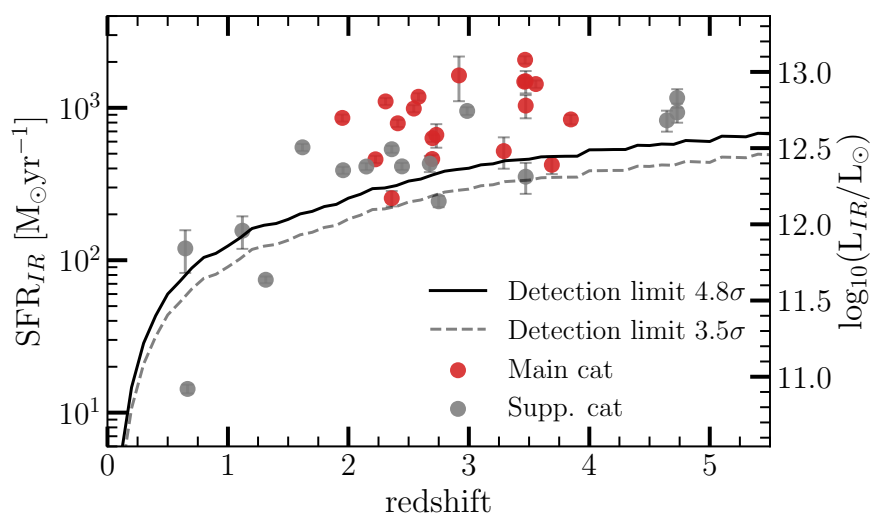

Fig. 3. Infrared star formation rate as a function of redshift, for galaxies from the main (red dots) and supplementary (gray dots) catalogs respectively. The SFR has been computed from the IR luminosity following Eq. (10). The detection limits at $4.8 \sigma$ (solid black line) and $3.5 \sigma$ (dashed black line) have also been computed from the IR library of Schreiber et al. (2018b), with a dust temperature evolving with redshift taking into account the average value of the rms at $0.182 \mathrm{mJy}$.

survey at the limit of $4.8 \sigma$ (solid black line) used to create the main catalog, assuming a constant $\mathrm{rms}(\mathrm{rms}=0.182 \mathrm{mJy})$ over the whole map, as well as the $3.5 \sigma$ (dashed black line) limit used to build the supplementary catalog. However, as the rms is not constant, and therefore may be lower at some points in the map, some galaxies (AGS21, for example) appear below this line.

We note that there is a galaxy (AGS36) that is clearly offset from the detection limit, with a SFR $\sim 15 M_{\odot} \mathrm{yr}^{-1}$. This galaxy is atypical, as it has the lowest redshift in our sample $(z=0.66$, the same redshift as AGS30) and it also hosts a powerful AGN with an X-ray luminosity $=1.39 \times 10^{43} \mathrm{erg} \mathrm{s}^{-1}$.

The SFR limit has been computed taking into account the main sequence SED from Schreiber et al. (2018b), with the temperature and the fraction of polycyclic aromatic hydrocarbon (PAH) emission evolving as a function of redshift. The IR luminosity was calculated by integrating the flux from the SED using Eq. (11), and was then converted into SFR using Eq. (10).

This IR luminosity limit allows us to detect galaxies down to an IR luminosity of $10^{12} L_{\odot}$ at redshift $z=1.5$, and down to $3 \times 10^{12} L_{\odot}$ at redshift $z=4$. In other words, for a MS galaxy, this allows us to detect galaxies with a minimum stellar mass of $2.5 \times 10^{11} M_{\odot}, 1.8 \times 10^{11} M_{\odot}$ and $1.5 \times 10^{11} M_{\odot}$ for redshifts $z=2, z=3$ and $z=4$ respectively, using Eq. (9) of Schreiber et al. (2015).

The majority of the galaxies detected in this ALMA survey are starbursts, or in the upper part of the MS (see Fig. 4). Among the galaxies for which we determined the SFR, 54\% of them have a $R_{\mathrm{SB}}\left(\mathrm{SFR} / \mathrm{SFR}_{\mathrm{MS}}\right)>3$ (see Table 2$)$.

Not surprisingly, the most IR luminous galaxies have been listed in the main catalog. However, we note the presence of a portion of galaxies from the supplementary catalog that are also among the most IR luminous galaxies. The size of the galaxies explains this behavior. The galaxies detected in the supplementary catalog generally have larger sizes than those in the main catalog (F20a). Even though the peak flux is fainter on average, the integrated flux can reach values close to those of the main catalog.

The vast majority $(86 \%)$ of the galaxies analyzed in this study can be classified as ultraluminous infrared galaxies (ULIRGs) with $12<\log _{10}\left(L_{\mathrm{IR}} / L_{\odot}\right)<13$. Only one galaxy has an infrared luminosity slightly above this threshold. All of the

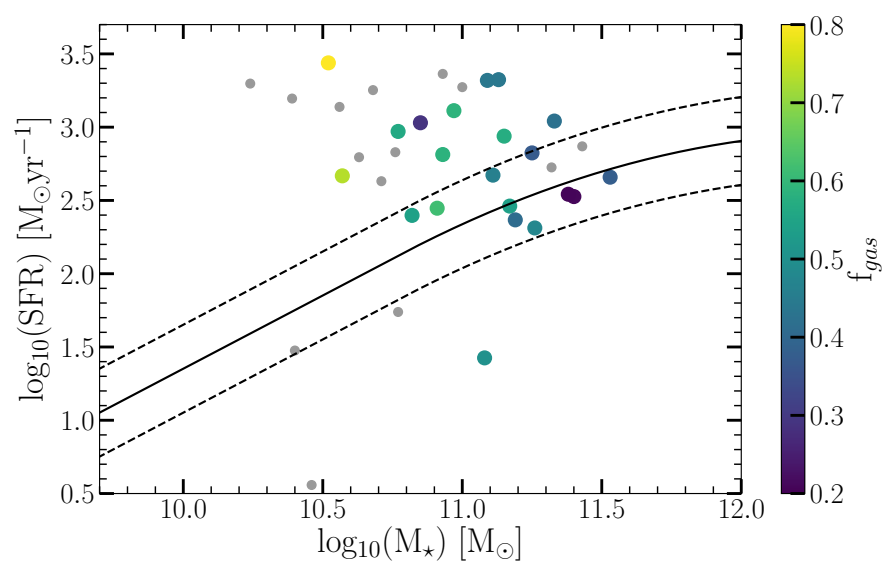

Fig. 4. Location of our ALMA detected galaxies in the SFR- $M_{\star}$ plane. Galaxies with Herschel counterparts are color-coded as a function of the $f_{\text {gas }}$. The other galaxies are represented by gray dots. We have rescaled all of the SFRs by multiplying by $\operatorname{SFR}_{\mathrm{MS}}(z) / \mathrm{SFR}_{\mathrm{MS}}(z=2.7)$, in order to maintain their relative positions with respect to the main sequence. We indicated the MS using Eq. (9) from Schreiber et al. (2015), with a dispersion of 0.3 dex (solid and dashed lines respectively).

galaxies with $\log _{10}\left(L_{\mathrm{IR}} / L_{\odot}\right)<12$ are galaxies less distant than the average of the galaxies detected in this survey, with $z<1.5$.

\subsection{2. $\mathrm{SFR}_{\mathrm{UV}}$}

Massive galaxies are known to be heavily dust-obscured at $z>2$ (e.g., Magnelli et al. 2009; Murphy et al. 2011). While the $S_{F R}{ }_{I R}$ is derived from the dust emission, we also consider the unobscured contribution to the total SFR, observed through UV emission. For the most massive galaxies $\left(M_{\star}>10^{10.5} M_{\odot}\right)$, the fraction of obscured to unobscured star formation $\left(\mathrm{SFR}_{\mathrm{IR}} / \mathrm{SFR}_{\mathrm{IR}+\mathrm{UV}}\right)$ is greater than 90\% (Whitaker et al. 2017).

We derive $L_{\mathrm{UV}}$ from the observed magnitude as follows:

$L_{\mathrm{UV}}\left[L_{\odot}\right]=\frac{4 \pi d_{\mathrm{L}}^{2} v_{1600} 10^{-0.4(48.6+m)}}{1+z}$

where $d_{\mathrm{L}}$ is the luminosity distance and $m$ is the observed magnitude. The $\mathrm{SFR}_{\mathrm{UV}}$, uncorrected for dust attenuation, is in turn derived from the $L_{\mathrm{UV}}$, following (Daddi et al. 2004):

$\mathrm{SFR}_{\mathrm{UV}}\left[M_{\odot} \mathrm{yr}^{-1}\right]=2.17 \times 10^{-10} \times L_{\mathrm{UV}}$

The total SFR $\left(\mathrm{SFR}_{\mathrm{tot}}=\mathrm{SFR}_{\mathrm{UV}}+\mathrm{SFR} \mathrm{R}_{\mathrm{IR}}\right)$ is given in Table 2 . Unless otherwise noted, in the following, SFR refers to the total SFR. The median contribution from $\mathrm{SFR}_{\mathrm{UV}}$ to $\mathrm{SFR}_{\mathrm{tot}}$ is $\sim 1 \%$.

\subsection{AGN}

Of the 1008 sources detected in X-ray during the 7 Ms exposure survey of the Chandra Deep Field-South presented in Luo et al. (2017), 397 lie in the GOODS-ALMA field. We adopted a cross-matching radius of $0.6^{\prime \prime}$, after applying the offset corrections presented in F20a. We found that 13/23 (6/20) of our main (supplementary) catalog galaxies had matches with the Luo et al. (2017) catalog. However, the detection in X-rays is not definitive proof that a galaxy hosts an AGN. We corrected the Luo et al. (2017) cataloged X-ray luminosities when redshift deviations were observed, using the following formula:

$L_{\mathrm{X}}=4 \pi d_{\mathrm{L}}^{2}(1+z)^{\Gamma-2} f_{\mathrm{X}}$ 
and assuming a fixed $\Gamma=2$. In the following paragraphs, a galaxy will be considered as hosting an AGN if the galaxy has an X-ray luminosity $L_{X, \text { int }}>10^{43} \mathrm{erg} \mathrm{s}^{-1}$ (luminous X-ray sources).

\section{The slow downfall of star formation in $z=2-3$ massive galaxies}

\subsection{A large fraction of galaxies in our sample with low gas fractions}

In this survey, we have detected particularly massive galaxies, the majority of which are beyond cosmic noon at $z \sim 1-2$. The study of the gas mass reservoirs is essential to understand how the galaxies will evolve with redshift and whether these galaxies could be the progenitors of passive galaxies at $z \sim 2$. To obtain the most robust results possible, we have considered in the following section only galaxies with a Herschel counterpart. The galaxies without a Herschel counterpart are marked with $\dagger$ in Table 2. In Fig. 5 (left panel), we compare the gas fraction of our galaxies as a function of their deviation from the MS, with the relationship presented in Tacconi et al. (2018):

$M_{\text {gas }} / M_{\star}=\left[0.66_{-23}^{+0.22}\right] \times R_{\mathrm{SB}}^{0.53}$.

In the same way, we compare the depletion time with the relationship presented in Tacconi et al. (2018):

$\tau_{\text {dep }}[\mathrm{Myr}]=\left[322_{-38}^{+43}\right] \times R_{\mathrm{SB}}^{-0.44}$

We have rescaled this relationship to correspond to the median redshift $\left(z_{\text {med }}=2.7\right)$ and the median stellar mass of our sample $\left(M_{\star, \text { med }}=8.5 \times 10^{10} M_{\odot}\right)$ by multiplying our results by $\tau_{\text {dep }}\left(z, M_{\star}\right) / \tau_{\text {dep }}\left(z_{\text {med }}, M_{\star \text {,med }}\right)$. To be able to directly compare the gas fraction of our galaxies to the relationship of Tacconi et al. (2018), we have also scaled our gas fractions according to the median redshift and stellar mass of our sample. The gas fractions, before rescaling, are presented in Table 2.

The depletion times span a large range, between 30 and 1600 Myr. The galaxies studied here show a dependence between depletion time and distance to the main sequence $\left(R_{\mathrm{SB}}\right)$, although very scattered (see Fig. 5).

About half of the GOODS-ALMA galaxies follow the $f_{\text {gas }}-R_{\mathrm{SB}}$ relation from Tacconi et al. (2018, Eq. (20)). However, we find a surprisingly large fraction $(40 \%)$ of galaxies lying well below this relation, that is, with excessively short depletion times (see Fig. 5). Galaxies lying below this relationship do not have a preferential redshift, we were detecting galaxies with redshifts close to the median redshift of our sample. On the other hand, the galaxies under this relation preferentially have stellar masses higher than the average of our sample with all but one exhibiting a stellar mass higher than $10^{11} M_{\odot}$. Moreover galaxies with a low gas fraction can either be on the main sequence or be starbursts.

The galaxies with the shortest depletion times are also those with the lowest gas fraction. This is because despite exhibiting lower gas masses, these galaxies keep forming stars with a high SFR.

We note that the majority of the ALMA galaxies experiencing a strong AGN episode with $L_{\mathrm{X}}>10^{43} \mathrm{erg} \mathrm{s}^{-1}$ lie below the $\tau_{\mathrm{dep}}-R_{\mathrm{SB}}$ and $f_{\mathrm{gas}}-R_{\mathrm{SB}}$ relations (stars in Fig. 5). This suggests that the low gas content and associated short depletion time of the galaxies may be due to the AGN feedback, heating the surrounding extragalactic medium and preventing further infall of gas. In other words, about half of the galaxies at these flux densities and redshifts appear to suffer from starvation and constitute excellent candidate progenitors of $z \simeq 2$ massive and compact elliptical galaxies. To further investigate this possibility, we show in Sect. 5.2.1 that the ALMA sizes, i.e., where the stars are formed, are consistent with the compact cores of $z=2$ elliptical galaxies.

However, there is a trend between $R_{\mathrm{SB}}$ and the stellar mass of the galaxies, in that the less massive galaxies in our sample have a larger average $R_{\mathrm{SB}}$. This is partly due to a selection effect. A deeper survey would be needed to investigate the population of galaxies on the main sequence with intermediate stellar masses. We also investigated the evolution of the depletion time as a function of the stellar mass but we found no correlation. This means that the star-formation efficiency $\left(\mathrm{SFE}=\mathrm{SFR} / M_{\mathrm{gas}}=1 / \tau_{\mathrm{dep}}\right)$ does not change according to the stellar mass of the galaxy.

The gas fractions cover a significant range of values, between $f_{\text {gas }}=0.21$ and 0.84 , with a median of $f_{\text {gas }}=0.52($ mean $=0.52)$. These values are consistent with other studies, such as Wiklind et al. (2014) for a sample with comparable stellar masses and redshifts. We do, however remark that for the two common galaxies between this work and Wiklind et al. (2014), there is a significant difference in the calculated gas fractions. These two common galaxies are outliers from the rest of the Wiklind et al. (2014) sample as they have gas fractions close to unity, and in fact, correspond to two HST-dark galaxies that were previously falsely attributed with optical counterparts.

We note that a significant number of the outliers with low gas fractions are classified as AGN. The presence of an AGN can influence the measurement of the stellar mass of the galaxy and artificially lower the calculated gas fraction of the galaxies. This result is consistent with the findings of Perna et al. (2018) who found systematically low gas fractions in obscured AGN at $z>1$ and suggests that AGN feedback could lead to the expulsion of gas. One of these galaxies has a low gas fraction (21\%) and does not show any sign of an AGN. This galaxy is a particularly striking example of interacting galaxies, with strong tidal tails. This galaxy does not have a high star formation rate, it lies on the MS, but it does display a starburst-like behavior since it exhibits a short gas depletion time. This galaxy could, therefore, be a member of the population of galaxies described in Elbaz et al. (2018), a starburst galaxy hidden in the main sequence.

We find a negative correlation between the stellar mass and the gas fraction (see Fig. 6, right panel). The following equation characterizes this relationship:

$f_{\text {gas }}=(-0.45 \pm 0.09) \times \log _{10}\left(M_{\star}\right)+5.44 \pm 0.95$.

A similar relationship has been found in other studies (e.g., Popping et al. 2012; Magdis et al. 2012; Sargent et al. 2014; Schinnerer et al. 2016). Galaxies hosting an AGN do not seem to occupy a particular position in Fig. 6. We also indicate in the left panel the distance to the main sequence as a function of the stellar mass. We can also see a clear negative correlation between the stellar mass and $R_{\mathrm{SB}}$. On the other hand, it is not possible to say whether selection effects are driving this trend. To be detected, a galaxy of low mass must have a larger $R_{\mathrm{SB}}$ than a massive galaxy. On the other hand, we do not find massive galaxies $\left(M_{\star}>10^{11} M_{\odot}\right.$ with $\left.R_{\mathrm{SB}}>5\right)$. We remain cautious in our interpretation of this result. As the size of our survey is modest, selection bias may be significant.

We found no correlation between the depletion time and the stellar mass. In our sample, the efficiency for the galaxies to transform their gas into stars is independent of the stellar mass of the galaxy. The Pearson's correlation coefficient between these two quantities is 0.05 , indicating the absence of a correlation. Galaxies with the lowest gas fractions also appear to be the most massive, suggesting that we are witnessing a slow downfall of 

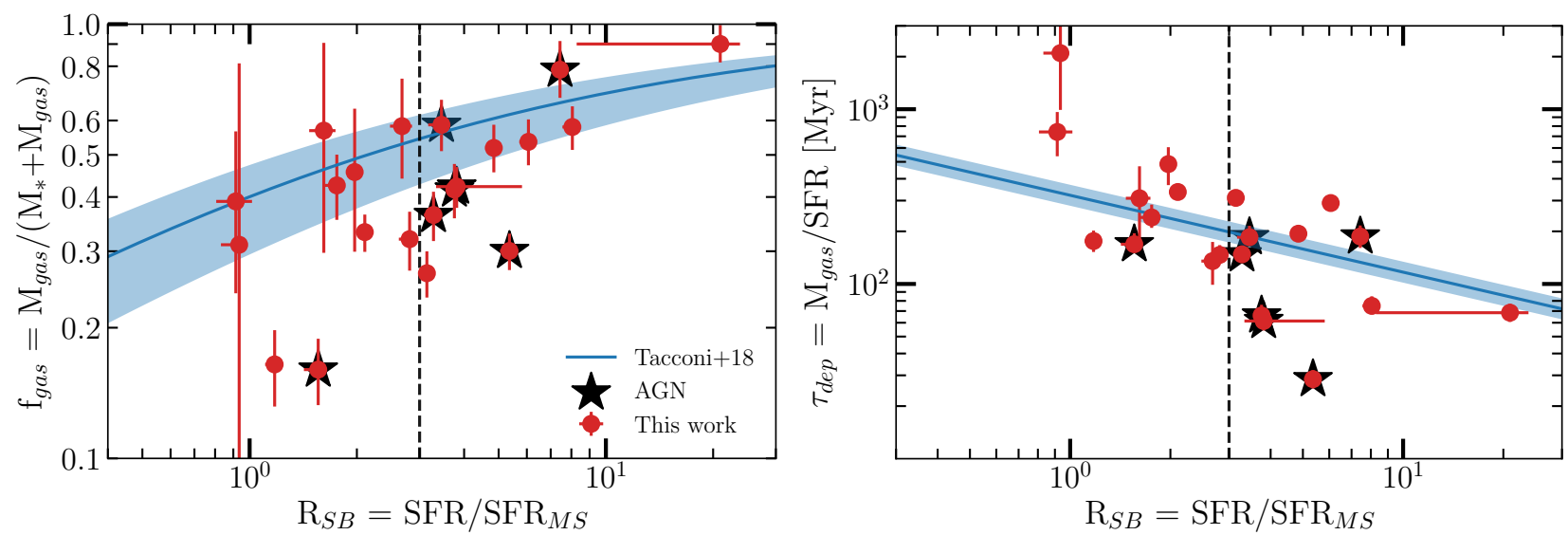

Fig. 5. Evolution of the molecular gas fraction $\left(f_{\mathrm{gas}}\right)$ and the gas depletion timescale $\left(\tau_{\mathrm{dep}}\right)$ as a function of the distance to the main sequence of star-forming galaxies $\left(R_{\mathrm{SB}}=\mathrm{SFR} / \mathrm{SFR} \mathrm{MS}_{\mathrm{MS}}\right)$ for the main and supplementary catalog of galaxies detected by ALMA in the GOODS-ALMA field. The solid blue line shows the relation obtained by Tacconi et al. (2018) for the median redshift and stellar mass of our sample $\left(z_{\mathrm{med}}=2.7\right.$, $\left.M_{\star \text { med }}=8.5 \times 10^{10} M_{\odot}\right)$. The uncertainty on the mean trend is obtained by Monte-Carlo simulations. In order to compare the gas fractions of all of the galaxies in our sample, we have rescaled our gas fractions according to the median redshift and the stellar mass of our sample.
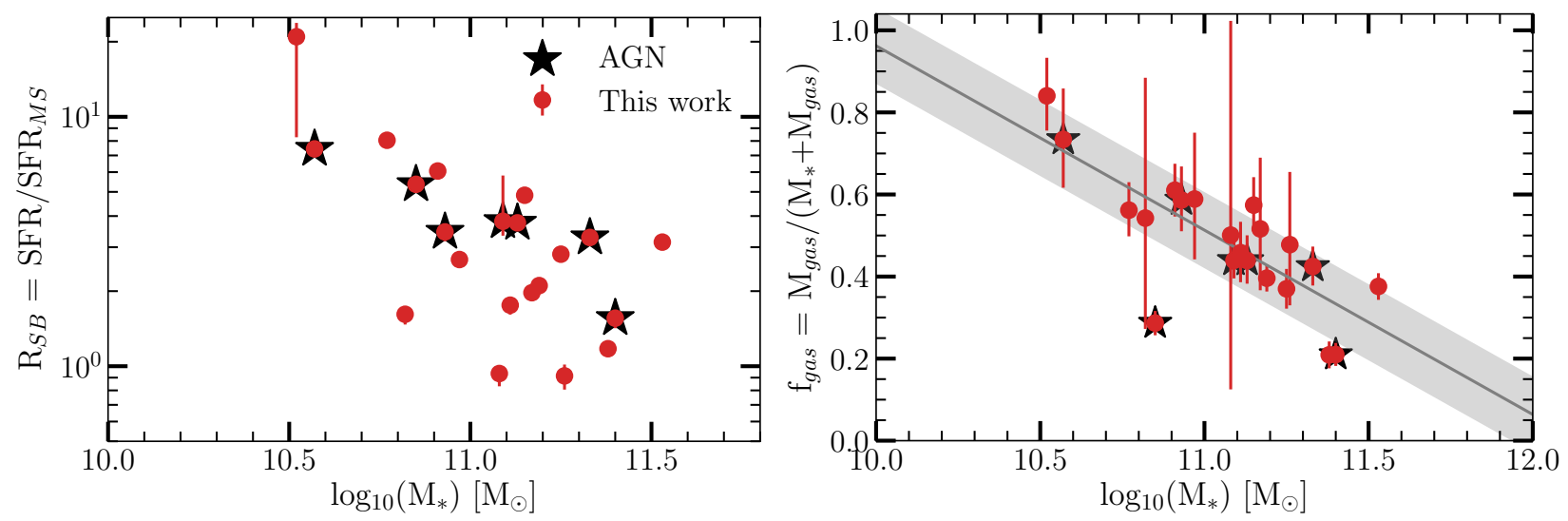

Fig. 6. Evolution of the distance to the main sequence of star-forming galaxies $\left(R_{\mathrm{SB}}=\mathrm{SFR} / \mathrm{SFR}_{\mathrm{MS}}\right.$, left panel $)$ and the molecular gas fraction $\left(f_{\text {gas }}\right.$, right panel) as a function of the stellar mass. The best fit, given by Eq. (17), is shown by the gray shaded region.

the galaxies with the most massive galaxies dying first to become elliptical galaxies, in a similar way to what has been shown in Schreiber et al. (2016), but at higher redshifts. This is consistent with the idea of "downsizing" where the most massive are also the ones that form their stars the earliest and fastest (e.g., Cowie et al. 1996; Guzmán et al. 1997; Brinchmann \& Ellis 2000; Neistein et al. 2006; Fontanot et al. 2009).

\subsection{Toward a reduction in the size of galaxies}

\subsubsection{Size}

Several studies have reported the observation of massive starforming galaxies, compact at a rest-frame wavelength of $5000 \AA$ or in the $H$-band (e.g., blue nuggets; Barro et al. 2013; Dekel \& Burkert 2014). It has been proposed that these galaxies are the progenitors of massive, compact and passive galaxies at $z=2$ (e.g., Barro et al. 2013, 2016; Williams et al. 2014; Toft et al. 2014; van der Wel et al. 2014; Kocevski et al. 2017).

We have, thanks to the GOODS-ALMA survey, selected a sample of massive star-forming galaxies. These galaxies are among the most massive ones within the UVJ active - in other words, star-forming - galaxies (Williams et al. 2009, using the same definition as in F18) listed in the ZFOURGE catalog (see
Fig. 10 in F20a). For example, with ALMA we have detected the most massive ZFOURGE galaxy in the redshift range $1<z<2$, the most massive galaxy at $2<z<3$, the second most massive galaxy at $3<z<4$. These galaxies cannot continue to form stars for long periods. If this were the case, they would become much more massive than the most massive galaxies we have observed at $z \sim 1$, or in the local universe.

The galaxies in the present paper have not been selected to be compact at a rest-frame wavelength of $5000 \AA$. They are fluxselected. Due to the low dispersion of the main sequence, this selection can be seen as a stellar mass selection. We aim to study here whether galaxies that have not been selected to be compact at $5000 \AA$ can also be the progenitors of compact galaxies at $z \simeq 2$. To do this, we have compared the $5000 \AA$ sizes of the galaxies detected by ALMA with the $5000 \AA$ sizes of the UVJ active galaxies located in the area defined by the GOODSALMA survey and detected during the CANDELS program.

The majority of the galaxies studied in this paper have a redshift between $z=2$ and 4 . We report in Fig. 7-left panel, the $5000 \AA$ sizes of all galaxies within $2<z<4$ located in the area defined by the GOODS-ALMA survey, as a function of stellar mass, in blue. We also show the $5000 \AA$ sizes of the ALMAdetected galaxies color-coded with redshift. Galaxy sizes and 
Sérsic indices are obtained from van der Wel et al. (2014). These values have been computed by fitting a single-component Sérsic profile using GALFIT (Peng et al. 2010) at both 1.25 and $1.6 \mu \mathrm{m}$. Following van der Wel et al. (2014), the effective radius at $5000 \AA$ has been estimated as:

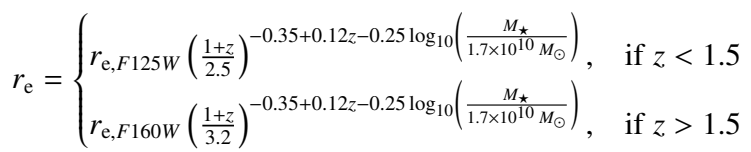

where $r_{\mathrm{e}, F 125 W}$ and $r_{\mathrm{e}, F 160 W}$ are the effective radius though the $F 125 \mathrm{~W}$ and $F 160 \mathrm{~W}$ filter respectively. We also show the trends for the UVJ active and UVJ passive galaxies with blue and red lines respectively. These two relations were parametrized by van der Wel et al. (2014) following:

$r_{\mathrm{e}}=A\left[\left(M_{\star} / 5 \times 10^{10}\right) / 1.7\right]^{\alpha}$,

where $r_{\mathrm{e}}$ is the effective radius, in other words, the semi-major axis of the ellipse that contains half of the total flux of the best-fitting Sérsic model, in kpc. We use the following parameters: $\log _{10}(A)=-0.06 \pm 0.03, \alpha=0.79 \pm 0.07$, and the scatter in $\left(r_{\mathrm{e}}\right)$ in logarithmic units $\sigma \log _{10}\left(r_{\mathrm{e}}\right)=0.14 \pm 0.03$ for earlytype galaxies and $\log _{10}(A)=0.51 \pm 0.01, \alpha=0.18 \pm 0.02$, and $\sigma \log _{10}\left(r_{\mathrm{e}}\right)=0.19 \pm 0.01$ for late-type galaxies (van der Wel et al. 2014).

We see that there is a significant difference in size between active and quiescent galaxies. The size of star-forming galaxies is on average larger than passive galaxies. Mosleh et al. (2011) noted, for example, that UV-bright galaxies with $10^{10}<M_{\star} / M_{\odot}<10^{11}$ and $0.5<z<3.5$ are larger than quiescent galaxies in the same mass and redshift range by $0.45 \pm 0.09$ dex.

For the vast majority of the ALMA detected galaxies, their optical rest-frame sizes are comparable to the $5000 \AA$ sizes of the UVJ active galaxies (blue hexagons) at $2<z<4$ selected in the same field of view. We also over-plot, in Fig. 7, the compactness criterion given in Barro et al. (2013) and modified by Barro et al. (2016):

$\Sigma_{1.5}=\frac{M_{\star}}{r_{\mathrm{e}}^{1.5}} \geq 10^{10.4} M_{\odot} \mathrm{kpc}^{-1.5}$.

This relation initially defined for $H$-band sizes was then rescaled according to Eq. (18) with a redshift equal to the median redshift of our sample of galaxies to make it correspond to a size at $5000 \AA$ A. Only three GOODS-ALMA galaxies are compact following the compactness criterion of Eq. (20). These galaxies lie on the trend for quiescent galaxies. We note that those galaxies that do not follow the trend of star-forming galaxies systematically host an AGN. If these galaxies suddenly stopped forming stars, they would already be located on the right trend in the mass-size diagram to be compact massive galaxies. With the data available to us, it is not possible to distinguish whether the compaction of the galaxy has triggered the AGN or, on the contrary, it is the presence of the AGN that has caused its compaction. We note that for galaxies with higher stellar mass $\left(M_{\star}>10^{11} M_{\odot}\right)$, the star-forming and quiescent size-mass relations converge making it more difficult to investigate these trends.

We also show the ALMA $1.1 \mathrm{~mm}$ sizes in comparison to the $5000 \AA$ sizes in Fig. 7, right panel. The ALMA sizes for the main and supplementary catalogs are given in F20a and in Table A.1. The size distribution differs slightly between the two samples. We showed in F18 that we were biased toward compact sources with our detection limit of $4.8 \sigma$. By lowering this detection threshold in the supplementary catalog, which was made possible as a result of basing our detections on IRAC and VLA detections, we are now detecting galaxies with larger ALMA sizes. For the 26 galaxies for which we have both HST $5000 \AA$ sizes and could measure a $1.1 \mathrm{~mm}$ size with ALMA, we find that ALMA sizes are generally smaller, with a median $r_{\mathrm{e}, \mathrm{HST}} / r_{\mathrm{e}, \mathrm{ALMA}}=2.3$. This ratio is significantly higher than the ratio of 1.4 found by Fujimoto et al. (2017) at $870 \mu \mathrm{m}$, for a sample of 1034 ALMA sources.

Considering that dust emission is a good indicator of dustobscured star formation, this result indicates that compact dustobscured star formation (at least more compact than optical emission) is taking place in the core of the galaxies studied here. This study confirms the comparison of optical and millimeter sizes performed at $z \simeq 1.3$ by Puglisi et al. (2019), and extends it to higher redshifts, at and before the epoch of the peak of cosmic star formation.

For these galaxies to be the progenitors of compact elliptical galaxies at $z \simeq 2$, they need to become more compact than their $5000 \AA$ size. The observed strong star formation activity concentrated in a small region of the galaxy can morphologically transform a galaxy into a more compact object. Assuming that there is no addition of gas, the majority of these galaxies have gas reservoirs equal to or close to their stellar mass. If this gas is transformed into stars in the compact emission region detected by ALMA, these galaxies will become compact and gradually migrate into the location of the mass-size diagram reserved for passive galaxies.

The ALMA galaxies presented here exhibit a present Sérsic index in the $H$-band of $\left\langle n_{\mathrm{AGS}}\right\rangle=1.63$. We have seen that the amount of star formation associated to the compact $1.1 \mathrm{~mm}$ emission is large enough to bring the half-light radius of the ALMA galaxies on top of the one expected for passive compact galaxies at $z \sim 2$, hence the question that remains to be answered is whether this evolution will also be accompanied with an increase of the Sérsic index that will bring them closer to the one observed for passive compact galaxies, i.e., increasing from $n=1.6$ to $n=2.6$. To answer this question, we would need to know with enough accuracy what is the actual Sérsic index of the ALMA sources in the $1.1 \mathrm{~mm}$ band. Unfortunately our resolution and depth are not sufficient to derive a Sérsic index for the dust emission, hence we cannot answer the question without supplementary information. We note however, that at least some of the ALMA sources may present Sérsic indices similar to those measured by Hodge et al. (2016), Elbaz et al. (2018) and Rujopakarn et al. (2019) who measured Sérsic indices close to $n \sim 1$ for galaxies with similar behavior in term of stellar mass, SFR and redshift. A simple model of the impact of the newly formed stars following such an index to the final stellar distribution of the galaxies suggests that they would remain below $n=2.6$. Hence we conclude that despite the fact that the ALMA galaxies will inevitably have compact final half-light radii, only a fraction of them will end up showing the high Sérsic index of the compact ellipticals observed at $z \sim 2$. We note that this index itself presents a distribution, hence we cannot reject the possibility that most of the present ALMA sources represent reliable progenitors of compact ellipticals at $z \sim 2$. This galaxy sample is building compact bulges. This is consistent with a scenario of rapid bulge growth in galaxies and that these bulges can already be in place at $z \sim 2$ (Tacchella et al. 2015).

\subsubsection{Morphology}

We here aim to look at the mechanisms that may have driven the gas in the center of the ALMA galaxies. This may be violent disk 

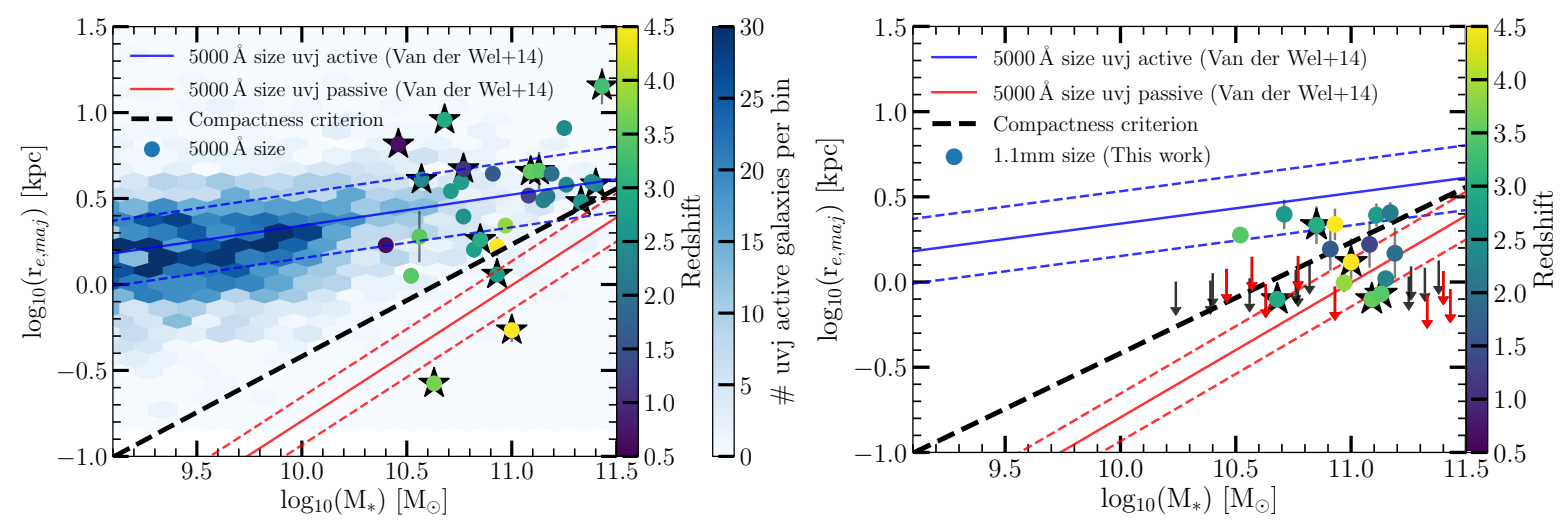

Fig. 7. Left panel: $5000 \AA$ A size-mass plane for the galaxies located in the GOODS-South field for which sizes have been measured in van der Wel et al. (2014). The density of the UVJ active galaxies (with $2<z<4$ ) in the GOODS-ALMA field is represented by the blue hexagons. The blue and red lines represent the trends of active and passive galaxies respectively, while the dashed lines give the scatter on these relations (van der Wel et al. 2014). The ALMA-detected galaxies are shown with dots color-coded by redshift. The stars represent galaxies with $L_{\mathrm{X}, \mathrm{int}}>10^{43} \mathrm{erg} \mathrm{s}^{-1}$. Right panel: ALMA size-mass plane for the ALMA detected galaxies. For comparison, the trends for active and passive galaxies are also shown. We indicate the compactness criterion described in Eq. (20) to visualize which galaxies are compact at $5000 \AA$ A. In this figure, ALMA sizes have been divided by a factor of $\sqrt{0.65}$, which corresponds to the median of the b/a ratio, to reflect size differences with HST. Galaxies with $L_{\mathrm{X}, \mathrm{int}}>10^{43} \mathrm{erg} \mathrm{s}^{-1}$ and for which only an upper limit in ALMA size has been determined are displayed with a red arrow.

instabilities (Dekel \& Burkert 2014), or other dissipative processes, including mergers (Wellons et al. 2015). To investigate the role of mergers in the compaction process, we now investigate the morphology of the ALMA-detected galaxies.

Increasing numbers of observations have demonstrated that elliptical galaxies at $z=2$ are particularly compact (e.g., Trujillo et al. 2006; van Dokkum et al. 2008; Conselice 2014; van der Wel et al. 2014). Major merger events can give rise to elliptical galaxies (e.g., Dekel \& Cox 2006; Hopkins et al. 2006a), but can also influence the compactness of the star formation in galaxies (e.g., Wuyts et al. 2010; Ceverino et al. 2015). Due to their large stellar masses, which has generated and retained a large amount of metals, and hence dust, against outflows (e.g., Dekel \& Silk 1986; Dekel \& Woo 2003; Tremonti et al. 2004), the galaxies detected in this study are extremely dust-obscured. In addition to this, their redshift makes them particularly faint in UV and optical filters. Some of them are $Y$-dropout (e.g., AGS5, AGS18), $V$-dropouts (e.g., AGS9, AGS10) or visible only in the $K s$-band (AGS4, AGS11, etc.). The morphology of these galaxies is therefore difficult to obtain. We cross-matched our sample with the catalog of Huertas-Company et al. (2015a) that estimates the probability of being a spheroid, disk or irregular using the Convolutional Neural Network technique. In addition to the 6 HST-dark galaxies, which, by definition, cannot be categorized, nine other galaxies have $H$-band fluxes too faint to be classified $(F 160 W>24.5 \mathrm{AB}$ mag). This leaves only 20 of our galaxies that are present in this catalog. We use the simplified classification proposed in Huertas-Company et al. (2015b):

- pure bulges: $f_{\text {sph }}>2 / 3$ AND $f_{\text {disk }}<2 / 3$ AND $f_{\text {irr }}<1 / 10$

- pure disks: $f_{\mathrm{sph}}<2 / 3$ AND $f_{\text {disk }}>2 / 3$ AND $f_{\text {irr }}<1 / 10$;

- disk + sph: $f_{\text {sph }}>2 / 3$ AND $f_{\text {disk }}>2 / 3$ AND $f_{\text {irr }}<1 / 10$;

- irregular disks: $f_{\text {disk }}>2 / 3$ AND $f_{\text {sph }}<2 / 3$ AND $f_{\text {irr }}>1 / 10$;

- irregulars/mergers: $f_{\text {disk }}<2 / 3$ AND $f_{\text {sph }}<2 / 3 \quad$ AND $f_{\text {irr }}>1 / 10$.

As a result, $61 \%(11 / 18)$ of our galaxies are classified as irregulars/mergers (two galaxies do not fit into any of the categories presented above). If we also take into account irregular disks, $78 \%(14 / 18)$ have an irregular morphology. Several galaxies show clear morphological characteristics of mergers, for example with large tidal tails. The galaxy AGS31, which exhibits large tidal tails, is an excellent illustration of this (see Appendix A in
F20a). For other galaxies, the interaction with another galaxy is more weak or uncertain.

We compared these proportions against those for a control sample. For each of the 18 galaxies in our sample with estimated morphologies from the Huertas-Company et al. (2015a) catalog, we selected the galaxy closest to it in terms of redshift and stellar mass, in the control sample. This control sample exhibits significantly different morphological proportions. Only $6 \%(1 / 18)$ of these galaxies can be classified as irregulars/mergers, $22 \%(4 / 18)$ if we take into account irregular disks. The galaxy population detected by ALMA, therefore, tends to be on average biased toward irregular galaxies. By more precisely considering the morphological classification, we obtain for the sample galaxies detected by ALMA an average $f_{\text {sph }}=0.16$, $f_{\text {disk }}=0.50, f_{\text {irr }}=0.34$, while for the control sample, an average of $f_{\text {sph }}=0.40, f_{\text {disk }}=0.53, f_{\text {irr }}=0.07$. While the disk fraction is relatively constant between these two samples, we are witnessing an inversion of the fraction between the irregulars and the spheroids. This result is consistent with a scenario in which a gas-rich major merger could funnel the gas into the center (e.g., Barnes \& Hernquist 1991; Hopkins et al. 2006b; Dekel \& Burkert 2014; Zolotov et al. 2015; Tacchella et al. 2016). This would explain the high fraction of irregular galaxies compared to the control sample.

We are therefore in the presence of a heterogeneous population of both secularly evolving disk and merger-type galaxies. The number of galaxies classified as irregulars/mergers is slightly higher with that found by models (Hayward et al. 2011, 2013), which predict that for a population of SMGs with $S_{1.1 \mathrm{~mm}}>0.5 \mathrm{mJy}$, star-forming galaxy-pairs account for $\sim 30-50 \%$ of the galaxies.

\subsection{IR surface brightness as a prior for the remaining lifetime of a galaxy}

The role of compact star formation in enhancing the efficiency of star formation is illustrated in Fig. 8. Galaxies forming stars with the largest star-formation surface density, $\Sigma_{\mathrm{SFR}}$, experience the strongest star formation episodes with the shortest depletion times (see Table 3).

The SFR surface density $\left(\Sigma_{\mathrm{SFR}}\right)$ can be defined as: 


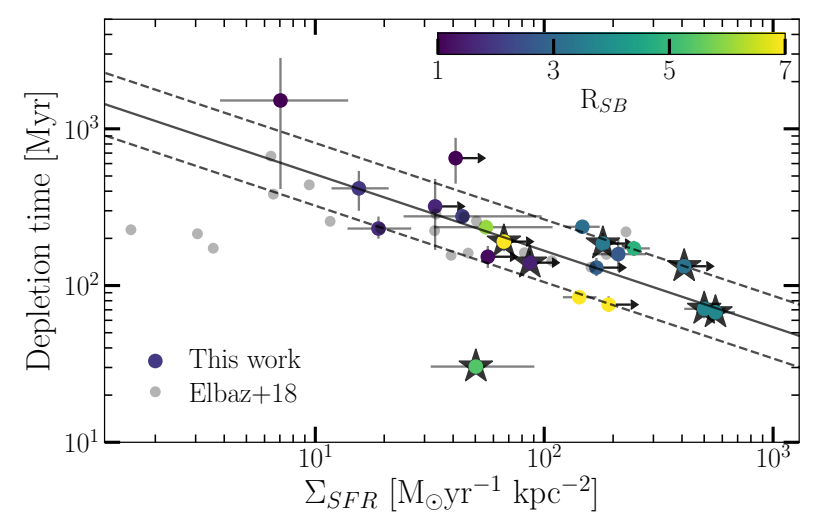

Fig. 8. Depletion time as a function of the $\Sigma_{\mathrm{SFR}}$, color-coded according to the distance to the main sequence. The solid and dashed lines are the fit to the sliding median and its $68 \%$ scatter respectively. The stars represent galaxies with $L_{\mathrm{X} \text {,int }}>10^{43} \mathrm{erg} \mathrm{s}^{-1}$. For comparison, the results of Elbaz et al. (2018) are shown by gray dots.

$\Sigma_{\mathrm{SFR}}=\operatorname{SFR} /\left(2 \pi R_{1.1 \mathrm{~mm}}^{2}\right)$,

where $R_{1.1 \mathrm{~mm}}$ is the half light radius (see Sect. 5.2.1 for a description of the determination of the millimeter size).

We have found a strong negative correlation between $\Sigma_{\mathrm{SFR}}$ and depletion time (see Fig. 8). A similar trend was found in Elbaz et al. (2018). This correlation can be characterized by the following equation:

$\tau_{\text {dep }}[\mathrm{Myr}]=10^{(3.20 \pm 0.25)} \times \Sigma_{\mathrm{SFR}}^{(-0.49 \pm 0.12)}$.

\section{Conclusions}

We have taken advantage of the excellent multiwavelength supporting data in the GOODS-South field and the largest contiguous ALMA survey to derive the physical properties of 35 ALMA flux-selected galaxies. This sample of galaxies comes both from a purely blind search (galaxies with a peak flux $>4.8 \sigma$, see F18) and from an extension of this catalog that we have built down to the $3.5 \sigma$ limit using IRAC and VLA to probe fainter millimeter galaxies (F20a). The number of galaxies comprising our sample is comparable to the expected number of galaxies reported in the number counts literature at similar flux and wavelengths to those of our study (e.g., Hatsukade et al. 2013, 2018; Oteo et al. 2016; Aravena et al. 2016; Umehata et al. 2017; Fujimoto et al. 2017; Dunlop et al. 2017; Franco et al. 2018; González-López et al. 2020; Popping et al. 2020) indicating that our sample of galaxies is almost complete.

These galaxies are massive $\left(M_{\star, \text { med }}=8.5 \times 10^{10} M_{\odot}\right)$ and therefore rare, so in order to be able to detect and analyze them, a sufficiently large survey, such as GOODS-ALMA was needed. It is possible now, for the first time with this survey, covering $\sim 69 \operatorname{arcmin}^{2}$. The analysis of the SEDs of these galaxies has made it possible to derive some of the physical properties of these galaxies. We are confronted with a heterogeneous population of galaxies. However, we highlight that about $40 \%$ of our galaxy sample exhibits a particularly small gas fraction. We remark that the most massive galaxies in our sample are also the galaxies with the lowest gas fractions. With their high star formation rates (the galaxies are mostly starbursts, or on the upper part of the main sequence) and without a gas refill mechanism, they will consume their gas reservoirs in a typical time of $100 \mathrm{Myr}$.

We also studied the sizes of these galaxies. The advantage of conducting a survey is that it does not impose a priori criteria
Table 3. Size, depletion time, and star-formation surface density.

\begin{tabular}{lccc}
\hline \hline ID & $\begin{array}{c}F W H M \\
(\operatorname{arcsec})\end{array}$ & $\begin{array}{c}\tau_{\text {dep }} \\
(\mathrm{Myr})\end{array}$ & $\begin{array}{c}\Sigma_{\text {SFR }} \\
\left(M_{\odot} \mathrm{yr}^{-1} \mathrm{kpc}^{-2}\right)\end{array}$ \\
\hline AGS1 & $0.21 \pm 0.02$ & $172_{-18}^{19}$ & $246_{-35}^{+42}$ \\
AGS3 & $<0.17$ & $132_{-14}^{+16}$ & $>408$ \\
AGS4 & $0.18 \pm 0.02$ & $67_{-7}^{+7}$ & $560_{-98}^{+117}$ \\
AGS5 & $0.19 \pm 0.02$ & $71_{-9}^{+9}$ & $500_{-93}^{+120}$ \\
AGS6 & $<0.19$ & $185_{-19}^{+29}$ & $>180$ \\
AGS8 & $0.23 \pm 0.02$ & $237_{-22}^{+24}$ & $146_{-23}^{+28}$ \\
AGS9 & $0.23 \pm 0.03$ & $158_{-36}^{+38}$ & $210_{-46}^{+66}$ \\
AGS10 & $<0.21$ & $130_{-15}^{+20}$ & $>169$ \\
AGS12 & $<0.23$ & $75_{-7}^{+10}$ & $>191$ \\
AGS13 & $<0.23$ & $139_{-15}^{+26}$ & $>86$ \\
AGS17 & $0.41 \pm 0.03$ & $84_{-7}^{+8}$ & $142_{-22}^{+27}$ \\
AGS18 & $0.50 \pm 0.08$ & $231_{-30}^{+44}$ & $18_{-5}^{+7}$ \\
AGS23 & $<0.24$ & $650_{-203}^{+222}$ & $>41$ \\
AGS26 & $0.30 \pm 0.09$ & $235_{-23}^{+26}$ & $55_{-22}^{+53}$ \\
AGS28 & $0.50 \pm 0.07$ & $416_{-117}^{+120}$ & $15_{-3}^{+5}$ \\
AGS29 & $<0.28$ & $447_{-85}^{+173}$ & $>19$ \\
AGS31 & $<0.27$ & $152_{-23}^{+25}$ & $>56$ \\
AGS34 & $<0.27$ & $319_{-149}^{+162}$ & $>33$ \\
AGS35 & $0.45 \pm 0.12$ & $30_{-3}^{+3}$ & $50_{-18}^{+38}$ \\
AGS37 & $0.28 \pm 0.10$ & $276_{-24}^{+27}$ & $43_{-19}^{+57}$ \\
AGS38 & $0.32 \pm 0.10$ & $1516_{-1103}^{+1256}$ & $7_{-3}^{+7}$ \\
AGS39 & $<0.28$ & $190_{-26}^{+29}$ & $>66$ \\
\hline & & &
\end{tabular}

Notes. Columns: (1) ALMA ID for galaxies with Herschel measurements; (2) FWHM measured from uvmodelfit in CASA; (3) depletion time $\left(\tau_{\text {dep }}=M_{\text {gas }} / \mathrm{SFR}\right)$, in Myr; (4) SFR surface density $\left(\Sigma_{\mathrm{SFR}}=\mathrm{SFR} /\left(2 \pi R_{1.1 \mathrm{~mm}}^{2}\right)\right)$, in $M_{\odot} \mathrm{yr}^{-1} \mathrm{kpc}^{-2}$.

for selecting the galaxies studied. The ALMA detected galaxies have observed $H$-band or $5000 \AA$ sizes comparable to the majority of galaxies with the same stellar masses and redshifts, whereas their dust emission regions, i.e., the regions tracing the obscured part of the star formation, are relatively compact and have sizes comparable to passive galaxies at $z \sim 2$.

We have investigated the link between depletion time and star formation surface density. We confirm the result showing a tight correlation between these two quantities. The denser the galaxy star-forming region is, the shorter the gas depletion time is. Mechanisms leading to a compaction of the obscured starforming regions are to be confirmed, but a compact region massively forming stars at the center of a galaxy can lead to a rapid morphological transition from a spiral to a compact elliptical galaxy such as those observed at $z \sim 2$, despite the fact that the ALMA selected galaxies are not yet compact at $5000 \AA$ or in the $H$-band (they are not yet blue nuggets).

All of these different pieces of evidence indicate that our ALMA-detected galaxies are the ideal progenitors of passive galaxies at $z \sim 2$ and natural exhaustion of their gas reservoirs (slow downfall) is sufficient for this transition to happen quickly without needing to invoke a ("fast") quenching mechanism. The large fraction of AGN among galaxies with the shortest depletion times and gas fractions suggest however that they may act by a starvation mechanism in preventing any further growth. 
Acknowledgements. We thank the anonymous referee for the insightful comments and suggestions that improved the clarity and quality of this work. M.F. acknowledges support from the UK Science and Technology Facilities Council (STFC) (grant number ST/R000905/1). B.M. acknowledges support from the Collaborative Research Centre 956, sub-project A1, funded by the Deutsche Forschungsgemeinschaft (DFG) - project ID 184018867. L.Z. acknowledges the support from the National Key R\&D Program of China (No. 2017YFA0402704, No. 2018YFA0404502), the National Natural Science Foundation of China (NSFC grants 11825302, 11733002 and 11773013) and China Scholarship Council (CSC). R.D. gratefully acknowledges support from the Chilean Centro de Excelencia en Astrofísica y Tecnologías Afines (CATA) BASAL grant AFB-170002. GEM acknowledges support from the Villum Fonden research grant 13160 "Gas to stars, stars to dust: tracing star formation across cosmic time", the Cosmic Dawn Center of Excellence funded by the Danish National Research Foundation and the ERC Consolidator Grant funding scheme (project ConTExt, grant number No. 648179). MP is supported by the ERC-StG "ClustersXCosmo", grant agreement 71676. DMA acknowledges support from the Science and Technology Facilities Council (ST/P000541/1; ST/T000244/1). This work was supported by the Programme National Cosmology et Galaxies (PNCG) of CNRS/INSU with INP and IN2P3, co-funded by CEA and CNES. This paper makes use of the following ALMA data: ADS/JAO.ALMA\#2015.1.00543.S. ALMA is a partnership of ESO (representing its member states), NSF (USA) and NINS (Japan), together with NRC (Canada), MOST and ASIAA (Taiwan), and KASI (Republic of Korea), in cooperation with the Republic of Chile. The Joint ALMA Observatory is operated by ESO, AUI/NRAO and NAOJ.

\section{References}

Álvarez-Márquez, J., Burgarella, D., Heinis, S., et al. 2016, A\&A, 587, A122 Aravena, M., Decarli, R., Walter, F., et al. 2016, ApJ, 833, 68 Ashby, M. L. N., Willner, S. P., Fazio, G. G., et al. 2013, ApJ, 769, 80 Baade, D., Meisenheimer, K., Iwert, O., et al. 1999, The Messenger, 95, 15 Barnes, J. E., \& Hernquist, L. E. 1991, ApJ, 370, L65 Barro, G., Faber, S. M., Pérez-González, P. G., et al. 2013, ApJ, 765, 104 Barro, G., Kriek, M., Pérez-González, P. G., et al. 2016, ApJ, 827, L32 Barro, G., Kriek, M., Pérez-González, P. G., et al. 2017, ApJ, 851, L40 Bell, E. F., McIntosh, D. H., Katz, N., \& Weinberg, M. D. 2003, ApJS, 149, 289 Berta, S., Lutz, D., Genzel, R., Förster-Schreiber, N. M., \& Tacconi, L. J. 2016, A\&A, 587, A73

Bian, F., Fan, X., Jiang, L., et al. 2013, ApJ, 774, 28

Blain, A. W., Smail, I., Ivison, R. J., Kneib, J.-P., \& Frayer, D. T. 2002, Phys. Rep., 369, 111

Boissier, S., Boselli, A., Buat, V., Donas, J., \& Milliard, B. 2004, A\&A, 424, 465 Boquien, M., Burgarella, D., Roehlly, Y., et al. 2019, A\&A, 622, A103 Bouwens, R. J., Illingworth, G. D., Oesch, P. A., et al. 2015, ApJ, 803, 34 Bouwens, R. J., Aravena, M., Decarli, R., et al. 2016, ApJ, 833, 72 Brinchmann, J., \& Ellis, R. S. 2000, ApJ, 536, L77 Bruzual, G., \& Charlot, S. 2003, MNRAS, 344, 1000

Calzetti, D., Armus, L., Bohlin, R. C., et al. 2000, ApJ, 533, 682 Caputi, K. I., Ilbert, O., Laigle, C., et al. 2015, ApJ, 810, 73 Cardamone, C. N., van Dokkum, P. G., Urry, C. M., et al. 2010, ApJS, 189, 270 Casey, C. M., Scoville, N. Z., Sanders, D. B., et al. 2014a, ApJ, 796, 95 Casey, C. M., Narayanan, D., \& Cooray, A. 2014b, Phys. Rep., 541, 45 Ceverino, D., Dekel, A., Tweed, D., \& Primack, J. 2015, MNRAS, 447, 3291 Chabrier, G. 2003, PASP, 115, 763

Chapman, S. C., Blain, A. W., Ivison, R. J., \& Smail, I. R. 2003, Nature, 422, 695

Ciesla, L., Elbaz, D., Schreiber, C., Daddi, E., \& Wang, T. 2018, A\&A, 615, A61 Conselice, C. J. 2014, ARA\&A, 52, 291

Coogan, R. T., Sargent, M. T., Daddi, E., et al. 2019, MNRAS, 485, 2092 Cowie, L. L., Songaila, A., Hu, E. M., \& Cohen, J. G. 1996, AJ, 112, 839 Cowie, L. L., González-López, J., Barger, A. J., et al. 2018, ApJ, 865, 106 Daddi, E., Cimatti, A., Renzini, A., et al. 2004, ApJ, 617, 746 Daddi, E., Bournaud, F., Walter, F., et al. 2010, ApJ, 713, 686 Dekel, A., \& Burkert, A. 2014, MNRAS, 438, 1870

Dekel, A., \& Cox, T. J. 2006, MNRAS, 370, 1445

Dekel, A., \& Silk, J. 1986, ApJ, 303, 39

Dekel, A., \& Woo, J. 2003, MNRAS, 344, 1131

Draine, B. T., Dale, D. A., Bendo, G., et al. 2007, ApJ, 663, 866 Draine, B. T., Aniano, G., Krause, O., et al. 2014, ApJ, 780, 172 Dunlop, J. S., McLure, R. J., Biggs, A. D., et al. 2017, MNRAS, 466, 861 Elbaz, D., Dickinson, M., Hwang, H. S., et al. 2011, A\&A, 533, A119 Elbaz, D., Leiton, R., Nagar, N., et al. 2018, A\&A, 616, A110 Fazio, G. G., Hora, J. L., Allen, L. E., et al. 2004, ApJS, 154, 10

Fontanot, F., De Lucia, G., Monaco, P., Somerville, R. S., \& Santini, P. 2009, MNRAS, 397, 1776
Franco, M., Elbaz, D., Béthermin, M., et al. 2018, A\&A, 620, A152 Franco, M., Elbaz, D., Zhou, L., et al. 2020, A\&A, in press, https: //doi .org/ 10. $1051 / 0004-6361 / 202038310$

Fu, H., Cooray, A., Feruglio, C., et al. 2013, Nature, 498, 338

Fujimoto, S., Ouchi, M., Ono, Y., et al. 2016, ApJS, 222, 1

Fujimoto, S., Ouchi, M., Shibuya, T., \& Nagai, H. 2017, ApJ, 850, 83 Fukugita, M., Hogan, C. J., \& Peebles, P. J. E. 1998, ApJ, 503, 518

Genzel, R., Tacconi, L. J., Combes, F., et al. 2012, ApJ, 746, 69

Giavalisco, M., Ferguson, H. C., Koekemoer, A. M., et al. 2004, ApJ, 600, L93

González-López, J., Decarli, R., Pavesi, R., et al. 2019, ApJ, 882, 139

González-López, J., Novak, M., Decarli, R., et al. 2020, ApJ, 897, 91

Gordon, K. D., Galliano, F., Hony, S., et al. 2010, A\&A, 518, L89

Griffin, M. J., Abergel, A., Abreu, A., et al. 2010, A\&A, 518, L3

Grogin, N. A., Kocevski, D. D., Faber, S. M., et al. 2011, ApJS, 197, 35

Guo, Y., Ferguson, H. C., Giavalisco, M., et al. 2013, ApJS, 207, 24

Guzmán, R., Gallego, J., Koo, D. C., et al. 1997, ApJ, 489, 559

Hatsukade, B., Ohta, K., Seko, A., Yabe, K., \& Akiyama, M. 2013, ApJ, 769, L27

Hatsukade, B., Kohno, K., Yamaguchi, Y., et al. 2018, PASJ, 70, 105

Hayward, C. C., Kereš, D., Jonsson, P., et al. 2011, ApJ, 743, 159

Hayward, C. C., Narayanan, D., Kereš, D., et al. 2013, MNRAS, 428, 2529

Hildebrandt, H., Erben, T., Dietrich, J. P., et al. 2006, A\&A, 452, 1121

Hodge, J. A., Karim, A., Smail, I., et al. 2013, ApJ, 768, 91

Hodge, J. A., Swinbank, A. M., Simpson, J. M., et al. 2016, ApJ, 833, 103

Hogg, D. W., \& Turner, E. L. 1998, PASP, 110, 727

Hopkins, P. F., Hernquist, L., Cox, T. J., et al. 2006a, ApJS, 163, 1

Hopkins, P. F., Hernquist, L., Cox, T. J., Robertson, B., \& Springel, V. 2006b, ApJS, 163, 50

Hsieh, B.-C., Wang, W.-H., Hsieh, C.-C., et al. 2012, ApJS, 203, 23

Huertas-Company, M., Gravet, R., Cabrera-Vives, G., et al. 2015a, ApJS, 221, 8

Huertas-Company, M., Pérez-González, P. G., Mei, S., et al. 2015b, ApJ, 809, 95

Hwang, H. S., Elbaz, D., Magdis, G., et al. 2010, MNRAS, 409, 75

Ivison, R. J., Papadopoulos, P. P., Smail, I., et al. 2011, MNRAS, 412, 1913

Kennicutt, R. C., Jr. 1998, ApJ, 498, 541

Kennicutt, R. C., Jr., \& Evans, N. J. 2012, ARA\&A, 50, 531

Kennicutt, R. C., Jr., Armus, L., Bendo, G., et al. 2003, PASP, 115, 928

Kewley, L. J., \& Dopita, M. A. 2002, ApJS, 142, 35

Kewley, L. J., \& Ellison, S. L. 2008, ApJ, 681, 1183

Kewley, L. J., Dopita, M. A., Leitherer, C., et al. 2013, ApJ, 774, 100

Kirkpatrick, A., Pope, A., Sajina, A., et al. 2015, ApJ, 814, 9

Kocevski, D. D., Barro, G., Faber, S. M., et al. 2017, ApJ, 846, 112

Koekemoer, A. M., Faber, S. M., Ferguson, H. C., et al. 2011, ApJS, 197, 36

Kovács, A., Chapman, S. C., Dowell, C. D., et al. 2006, ApJ, 650, 592

Leroy, A. K., Walter, F., Brinks, E., et al. 2008, AJ, 136, 2782

Leroy, A. K., Bolatto, A., Gordon, K., et al. 2011, ApJ, 737, 12

Liu, D., Daddi, E., Dickinson, M., et al. 2018, ApJ, 853, 172

Luo, B., Brandt, W. N., Xue, Y. Q., et al. 2017, ApJS, 228, 2

Madau, P., \& Dickinson, M. 2014, ARA\&A, 52, 415

Magdis, G. E., Elbaz, D., Hwang, H. S., et al. 2010, MNRAS, 409, 22

Magdis, G. E., Daddi, E., Elbaz, D., et al. 2011, ApJ, 740, L15

Magdis, G. E., Daddi, E., Béthermin, M., et al. 2012, ApJ, 760, 6

Magdis, G. E., Rigopoulou, D., Daddi, E., et al. 2017, A\&A, 603, A93

Magnelli, B., Elbaz, D., Chary, R. R., et al. 2009, A\&A, 496, 57

Mannucci, F., Cresci, G., Maiolino, R., Marconi, A., \& Gnerucci, A. 2010, MNRAS, 408, 2115

McAlpine, S., Smail, I., Bower, R. G., et al. 2019, MNRAS, 488, 2440

McLeod, D. J., McLure, R. J., Dunlop, J. S., et al. 2015, MNRAS, 450, 3032

Michałowski, M., Hjorth, J., \& Watson, D. 2010, A\&A, 514, A67

Miettinen, O., Delvecchio, I., Smolčić, V., et al. 2017, A\&A, 606, A17

Mosleh, M., Williams, R. J., Franx, M., \& Kriek, M. 2011, ApJ, 727, 5

Mullaney, J. R., Alexander, D. M., Goulding, A. D., \& Hickox, R. C. 2011, MNRAS, 414, 1082

Murphy, E. J., Condon, J. J., Schinnerer, E., et al. 2011, ApJ, 737, 67

Muzzin, A., Marchesini, D., Stefanon, M., et al. 2013, ApJ, 777, 18

Neistein, E., van den Bosch, F. C., \& Dekel, A. 2006, MNRAS, 372, 933

Noeske, K. G., Weiner, B. J., Faber, S. M., et al. 2007, ApJ, 660, L43

Nonino, M., Dickinson, M., Rosati, P., et al. 2009, ApJS, 183, 244

Nordon, R., Lutz, D., Shao, L., et al. 2010, A\&A, 518, L24

Obreschkow, D., \& Rawlings, S. 2009, MNRAS, 400, 665

Oesch, P. A., Bouwens, R. J., Illingworth, G. D., et al. 2015, ApJ, 808, 104

Oke, J. B., \& Gunn, J. E. 1983, ApJ, 266, 713

Oteo, I., Zwaan, M. A., Ivison, R. J., Smail, I., \& Biggs, A. D. 2016, ApJ, 822, 36

Pannella, M., Carilli, C. L., Daddi, E., et al. 2009, ApJ, 698, L116

Peng, C. Y., Ho, L. C., Impey, C. D., \& Rix, H.-W. 2010, AJ, 139, 2097

Perna, M., Sargent, M. T., Brusa, M., et al. 2018, A\&A, 619, A90 
Pettini, M., \& Pagel, B. E. J. 2004, MNRAS, 348, L59

Poglitsch, A., Waelkens, C., Geis, N., et al. 2010, A\&A, 518, L2

Popping, G., Caputi, K. I., Somerville, R. S., \& Trager, S. C. 2012, MNRAS, 425,2386

Popping, G., Walter, F., Behroozi, P., et al. 2020, ApJ, 891, 135

Puglisi, A., Daddi, E., Liu, D., et al. 2019, ApJ, 877, L23

Reddy, N. A. 2006, PhD Thesis, California Institute of Technology, USA

Reddy, N. A., Erb, D. K., Pettini, M., Steidel, C. C., \& Shapley, A. E. 2010, ApJ 712,1070

Ricciardelli, E., Trujillo, I., Buitrago, F., \& Conselice, C. J. 2010, MNRAS, 406, 230

Riechers, D. A., Bradford, C. M., Clements, D. L., et al. 2013, Nature, 496, 329

Rieke, G. H., Young, E. T., Engelbracht, C. W., et al. 2004, ApJS, 154, 25

Rodighiero, G., Daddi, E., Baronchelli, I., et al. 2011, ApJ, 739, L40

Rujopakarn, W., Dunlop, J. S., Rieke, G. H., et al. 2016, ApJ, 833, 12

Rujopakarn, W., Daddi, E., Rieke, G. H., et al. 2019, ApJ, 882, 107

Salpeter, E. E. 1955, ApJ, 121, 161

Santini, P., Maiolino, R., Magnelli, B., et al. 2014, A\&A, 562, A30

Sargent, M. T., Daddi, E., Béthermin, M., et al. 2014, ApJ, 793, 19

Schinnerer, E., Groves, B., Sargent, M. T., et al. 2016, ApJ, 833, 112

Schreiber, C., Pannella, M., Elbaz, D., et al. 2015, A\&A, 575, A74

Schreiber, C., Elbaz, D., Pannella, M., et al. 2016, A\&A, 589, A35

Schreiber, C., Labbé, I., Glazebrook, K., et al. 2018a, A\&A, 611, A22

Schreiber, C., Elbaz, D., Pannella, M., et al. 2018b, A\&A, 609, A30

Scoville, N., Sheth, K., Aussel, H., et al. 2016, ApJ, 820, 83

Seko, A., Ohta, K., Yabe, K., et al. 2016, ApJ, 833, 53

Silverman, J. D., Daddi, E., Rodighiero, G., et al. 2015, ApJ, 812, L23

Somerville, R. S., \& Davé, R. 2015, ARA\&A, 53, 51

Steidel, C. C., Adelberger, K. L., Giavalisco, M., Dickinson, M., \& Pettini, M. 1999, ApJ, 519, 1

Straatman, C. M. S., Spitler, L. R., Quadri, R. F., et al. 2016, ApJ, 830, 51

Swinbank, A. M., Chapman, S. C., Smail, I., et al. 2006, MNRAS, 371, 465

Swinbank, A. M., Simpson, J. M., Smail, I., et al. 2014, MNRAS, 438, 1267

Tacchella, S., Carollo, C. M., Renzini, A., et al. 2015, Science, 348, 314

Tacchella, S., Dekel, A., Carollo, C. M., et al. 2016, MNRAS, 458, 242

Tacconi, L. J., Genzel, R., Saintonge, A., et al. 2018, ApJ, 853, 179

Talia, M., Pozzi, F., Vallini, L., et al. 2018, MNRAS, 476, 3956

Tan, Q., Daddi, E., Sargent, M., et al. 2013, ApJ, 776, L24

Tan, Q., Daddi, E., Magdis, G., et al. 2014, A\&A, 569, A98

Toft, S., Smolčić, V., Magnelli, B., et al. 2014, ApJ, 782, 68

Tremonti, C. A., Heckman, T. M., Kauffmann, G., et al. 2004, ApJ, 613, 898

Trujillo, I., Förster Schreiber, N. M., Rudnick, G., et al. 2006, ApJ, 650, 18

Umehata, H., Tamura, Y., Kohno, K., et al. 2017, ApJ, 835, 98

van der Wel, A., Franx, M., van Dokkum, P. G., et al. 2014, ApJ, 788, 28

van Dokkum, P. G., Quadri, R., Marchesini, D., et al. 2006, ApJ, 638, L59

van Dokkum, P. G., Franx, M., Kriek, M., et al. 2008, ApJ, 677, L5

Walter, F., Decarli, R., Aravena, M., et al. 2016, ApJ, 833, 67

Wang, T., Elbaz, D., Schreiber, C., et al. 2016, ApJ, 816, 84

Wang, T., Schreiber, C., Elbaz, D., et al. 2019, Nature, 572, 211

Wardlow, J. L., Smail, I., Coppin, K. E. K., et al. 2011, MNRAS, 415, 1479

Wellons, S., Torrey, P., Ma, C.-P., et al. 2015, MNRAS, 449, 361

Whitaker, K. E., Pope, A., Cybulski, R., et al. 2017, ApJ, 850, 208

Wiklind, T., Conselice, C. J., Dahlen, T., et al. 2014, ApJ, 785, 111

Williams, R. J., Quadri, R. F., Franx, M., van Dokkum, P., \& Labbé, I. 2009 ApJ, 691, 1879

Williams, C. C., Giavalisco, M., Cassata, P., et al. 2014, ApJ, 780, 1

Williams, C. C., Labbe, I., Spilker, J., et al. 2019, ApJ, 884, 154

Wuyts, S., Labbé, I., Förster Schreiber, N. M., et al. 2008, ApJ, 682, 985

Wuyts, S., Cox, T. J., Hayward, C. C., et al. 2010, ApJ, 722, 1666

Yun, M. S., Reddy, N. A., \& Condon, J. J. 2001, ApJ, 554, 803

Zhou, L., Elbaz, D., Franco, M., et al. 2020, A\&A, 642, A155

Zolotov, A., Dekel, A., Mandelker, N., et al. 2015, MNRAS, 450, 2327

1 AIM, CEA, CNRS, Université Paris-Saclay, Université Paris Diderot, Sorbonne Paris Cité, 91191 Gif-sur-Yvette, France

2 Centre for Astrophysics Research, University of Hertfordshire, Hatfield AL10 9AB, UK

e-mail: m.franco@herts.ac.uk

3 School of Astronomy and Space Science, Nanjing University, Nanjing 210093, PR China

4 Key Laboratory of Modern Astronomy and Astrophysics (Nanjing University), Ministry of Education, Nanjing 210093, PR China

5 Argelander-Institut für Astronomie, Universität Bonn, Auf dem Hügel 71, 53121 Bonn, Germany
${ }^{6}$ Department of Physics, University of Oxford, Keble Road, Oxford OX1 3RH, UK

7 Aix Marseille Université, CNRS, LAM, Laboratoire d'Astrophysique de Marseille, Marseille, France

8 Community Science and Data Center/NSF's NOIRLab, $950 \mathrm{~N}$. Cherry Ave., Tucson, AZ 85719, USA

9 Department of Astronomy, Universidad de Concepción, Casilla 160-C, Concepción, Chile

10 Departamento de Astronomía, Facultad de Ciencias Físicas y Matemáticas, Universidad de Concepción, Concepción, Chile

11 Cosmic Dawn Center at the Niels Bohr Institute, University of Copenhagen and DTU-Space, Technical University of Denmark, Kgs. Lyngby, Denmark

12 DTU Space, National Space Institute, Technical University of Denmark, Elektrovej 327, 2800 Kgs. Lyngby, Denmark

13 Niels Bohr Institute, University of Copenhagen, 2100 Copenhagen, Denmark

14 Institute for Astronomy, Astrophysics, Space Applications and Remote Sensing, National Observatory of Athens, 15236 Athens, Greece

15 Centre for Extragalactic Astronomy, Department of Physics, Durham University, Durham DH1 3LE, UK

16 Institute of Astronomy, University of Tokyo, 2-21-1 Osawa, Mitaka, Tokyo 181-0015, Japan

17 Department of Physics and Astronomy, The University of Sheffield, Hounsfield Road, Sheffield S3 7RH, UK

18 Astronomy Centre, Department of Physics and Astronomy, University of Sussex, Brighton BN1 9QH, UK

19 Univ. Lyon, Univ. Lyon1, ENS de Lyon, CNRS, Centre de Recherche Astrophysique de Lyon (CRAL) UMR5574, 69230 Saint-Genis-Laval, France

20 Hiroshima Astrophysical Science Center, Hiroshima University, 1-3-1 Kagamiyama, Higashi-Hiroshima, Hiroshima 739-8526, Japan

21 Department of Physics, Anhui Normal University, Wuhu, Anhui 241000, PR China

22 Infrared Processing and Analysis Center, MS314-6, California Institute of Technology, Pasadena, CA 91125, USA

23 Max-Planck-Institut für Extraterrestrische Physik (MPE), Giessenbachstr.1, 85748 Garching, Germany

${ }^{24}$ Space Telescope Science Institute, 3700 San Martin Drive, Baltimore, MD 21218, USA

25 Department of Astronomy, The University of Texas at Austin, Austin, TX 78712, USA

26 Astronomy Department, University of Massachusetts, Amherst, MA 01003, USA

27 National Astronomical Observatory of Japan, National Institutes of Natural Sciences, 2-21-1 Osawa, Mitaka, Tokyo 181-8588, Japan

28 SOKENDAI (The Graduate University for Advanced Studies), 2-21-1 Osawa, Mitaka, Tokyo 181-8588, Japan

29 Institute of Astronomy \& Astrophysics, Academia Sinica, Taipei 10617, Taiwan

30 Institute of Astronomy, Graduate School of Science, The University of Tokyo, 2-21-1 Osawa, Mitaka, Tokyo 181-0015, Japan

31 Astronomy Unit, Department of Physics, University of Trieste, Via Tiepolo 11, 34131 Trieste, Italy

32 Fakultät für Physik der Ludwig-Maximilians-Universität, 81679 München, Germany

33 Department of Physics and Astronomy, Texas A\&M University, College Station, TX 77843-4242, USA

${ }^{34}$ George P. and Cynthia Woods Mitchell Institute for Fundamental Physics and Astronomy, Texas A\&M University, College Station, TX 77843-4242, USA

35 Department of Physics, Faculty of Science, Chulalongkorn University, 254 Phayathai Road, Pathumwan, Bangkok 10330, Thailand

36 National Astronomical Research Institute of Thailand (Public Organization), Donkaew, Maerim, Chiangmai 50180, Thailand

37 Kavli Institute for the Physics and Mathematics of the Universe (WPI), The University of Tokyo Institutes for Advanced Study, The University of Tokyo, Kashiwa, Chiba 277-8583, Japan 


\section{Appendix A: SEDs}
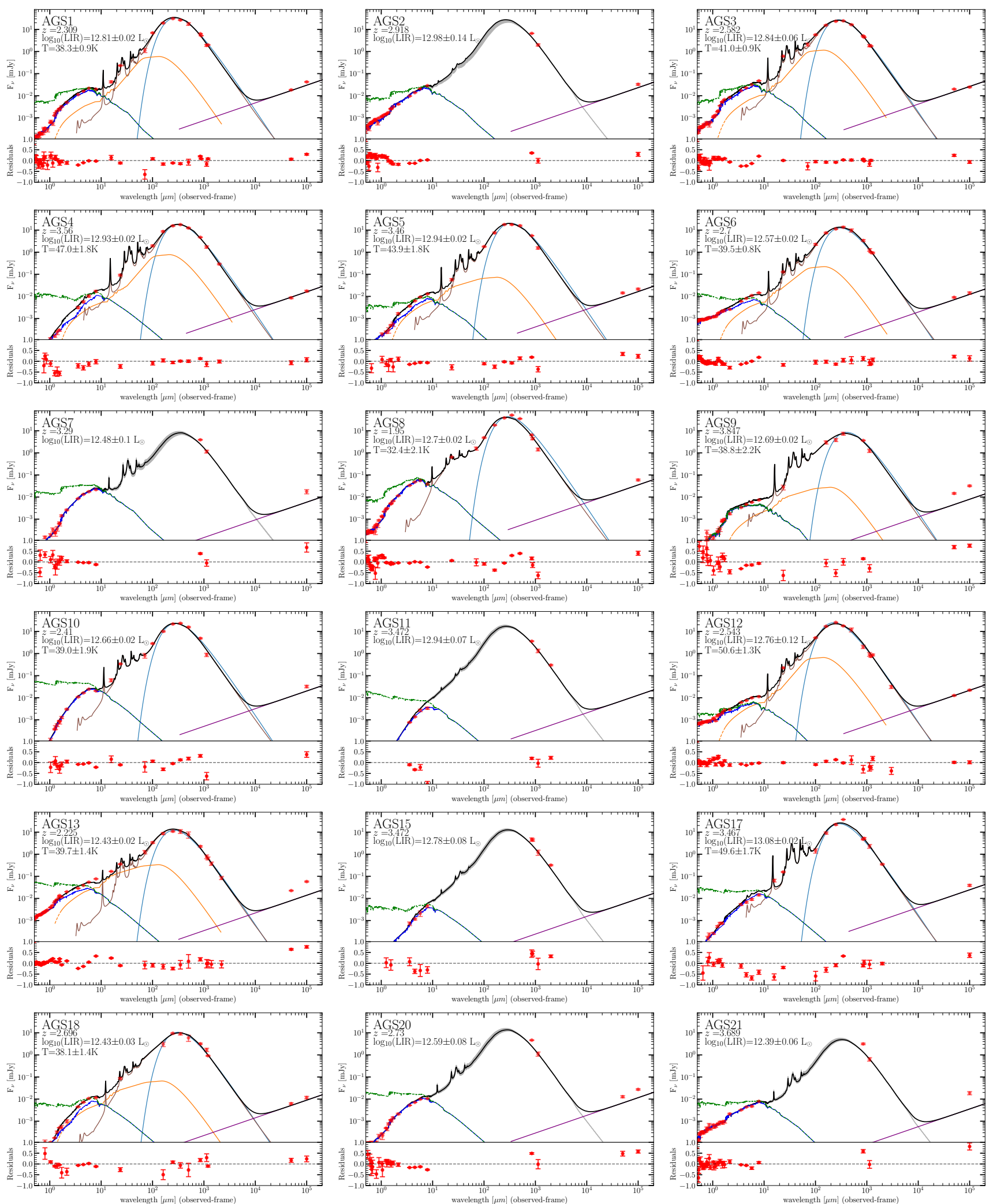

Fig. A.1. Optical to radio Spectral Energy Distributions for the 35 galaxies detected in the GOODS-ALMA survey. If the studied galaxy has also been detected with Hershel, we fit the SED using the CIGALE code, otherwise we use the dust spectral energy distribution library presented in Schreiber et al. (2018b). The solid black line represents the best fit, which can be decomposed into the IR dust contribution (brown line), a stellar component uncorrected for dust attenuation (dark blue line), synchrotron emission (purple line) and the AGN contribution (orange line). In addition, we show the best fit of a modified black body, with $\beta=1.5$ (light blue line). The corrected UV emission is also shown in green. The bottom panel shows the residuals: (observation - model)/observation. 

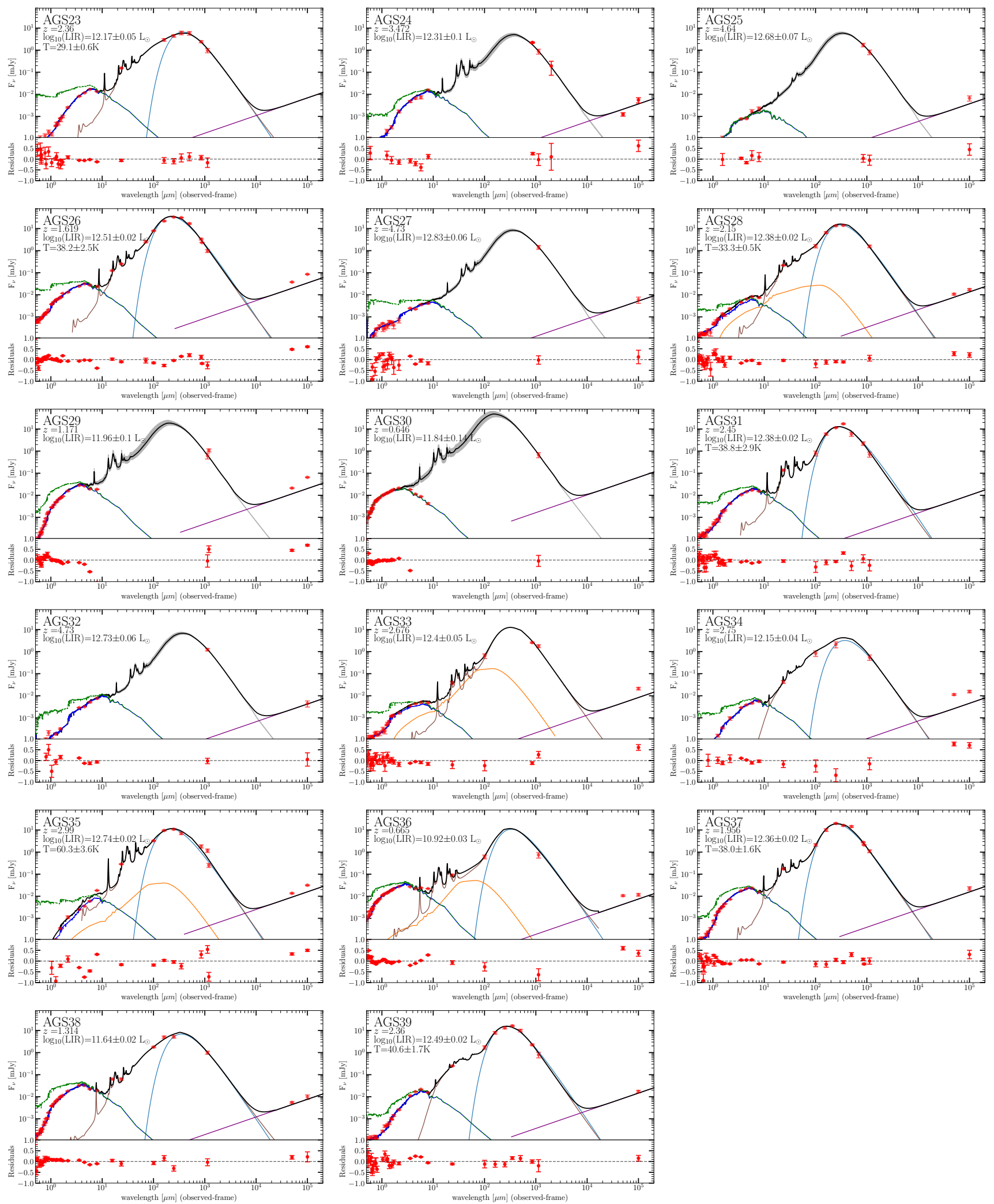

Fig. A.1. continued. 
M. Franco et al.: GOODS-ALMA: The slow downfall of star formation in $z=2-3$ massive galaxies

Table A.1. Summary of fluxes (peak flux using Blobcat and integrated flux using uvmodelfit), as well as the sizes (measured using uvmodelfit) for the galaxies in the Main and Supplementary catalogs.

\begin{tabular}{|c|c|c|c|}
\hline ID & $\begin{array}{c}S_{\text {peak }}^{\text {Blobcat }} \\
(\mathrm{mJy})\end{array}$ & 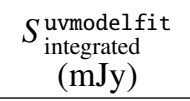 & $\begin{array}{c}F H W M^{\text {uvmodelfit }} \\
(\operatorname{arcsec})\end{array}$ \\
\hline AGS1 & $1.90 \pm 0.20 *$ & $2.20 \pm 0.13$ & $0.21 \pm 0.02$ \\
\hline AGS2 & $1.99 \pm 0.22 *$ & $2.31 \pm 0.12$ & $0.16 \pm 0.01$ \\
\hline AGS3 & $1.84 \pm 0.21 *$ & $1.97 \pm 0.12$ & $0.16 \pm 0.16$ \\
\hline AGS4 & $1.72 \pm 0.20 *$ & $1.68 \pm 0.11$ & $0.18 \pm 0.02$ \\
\hline AGS5 & $1.56 \pm 0.19 *$ & $2.49 \pm 0.18$ & $0.19 \pm 0.02$ \\
\hline AGS6 & $1.27 \pm 0.18^{*}$ & $1.37 \pm 0.13$ & $0.11 \pm 0.03$ \\
\hline AGS7 & $1.15 \pm 0.17^{*}$ & $1.64 \pm 0.11$ & $0.10 \pm 0.02$ \\
\hline AGS8 & $1.43 \pm 0.22 *$ & $2.23 \pm 0.17$ & $0.23 \pm 0.02$ \\
\hline AGS9 & $1.25 \pm 0.21 *$ & $1.70 \pm 0.18$ & $0.23 \pm 0.03$ \\
\hline AGS10 & $0.88 \pm 0.15^{*}$ & $1.18 \pm 0.13$ & $0.14 \pm 0.03$ \\
\hline AGS11 & $1.34 \pm 0.25 *$ & $1.71 \pm 0.17$ & $0.12 \pm 0.02$ \\
\hline AGS12 & $0.93 \pm 0.18^{*}$ & $1.17 \pm 0.15$ & $0.22 \pm 0.04$ \\
\hline AGS13 & $0.78 \pm 0.15^{*}$ & $0.84 \pm 0.11$ & $0.17 \pm 0.03$ \\
\hline AGS15 & $0.80 \pm 0.16$ & $1.21 \pm 0.11 *$ & $0.07 \pm 0.02$ \\
\hline AGS17 & $0.93 \pm 0.19$ & $2.30 \pm 0.20 *$ & $0.41 \pm 0.03$ \\
\hline AGS18 & $0.85 \pm 0.18$ & $1.70 \pm 0.28 *$ & $0.50 \pm 0.08$ \\
\hline AGS20 & $1.11 \pm 0.24^{*}$ & $1.90 \pm 0.20$ & $0.19 \pm 0.02$ \\
\hline AGS21 & $0.64 \pm 0.11^{*}$ & $0.75 \pm 0.12$ & $0.09 \pm 0.04$ \\
\hline AGS23 & $0.98 \pm 0.21 *$ & $0.94 \pm 0.16$ & $0.22 \pm 0.04$ \\
\hline AGS24 & $0.88 \pm 0.22 *$ & $0.81 \pm 0.29$ & $0.06 \pm 0.55$ \\
\hline AGS25 & $0.81 \pm 0.19 *$ & $1.06 \pm 0.28$ & $0.12 \pm 0.24$ \\
\hline AGS26 & $0.74 \pm 0.18$ & $0.97 \pm 0.15^{*}$ & $0.30 \pm 0.09$ \\
\hline AGS27 & $0.82 \pm 0.22$ & $1.43 \pm 0.28 *$ & $0.54 \pm 0.12$ \\
\hline AGS28 & $0.85 \pm 0.21$ & $1.56 \pm 0.21 *$ & $0.50 \pm 0.07$ \\
\hline AGS29 & $0.61 \pm 0.18^{*}$ & $1.22 \pm 0.19$ & $\ldots$ \\
\hline AGS30 & $0.67 \pm 0.17 *$ & $0.83 \pm 0.23$ & $\ldots$ \\
\hline AGS31 & $0.72 \pm 0.19 *$ & $1.01 \pm 0.17$ & \\
\hline AGS32 & $0.63 \pm 0.16$ & $1.23 \pm 0.16^{*}$ & $0.33 \pm 0.10$ \\
\hline AGS33 & $0.70 \pm 0.19$ & $1.77 \pm 0.27 *$ & $0.51 \pm 0.10$ \\
\hline AGS34 & $0.55 \pm 0.15^{*}$ & & \\
\hline AGS35 & $0.65 \pm 0.18$ & $1.16 \pm 0.21 *$ & $0.45 \pm 0.12$ \\
\hline AGS36 & $0.74 \pm 0.21 *$ & $0.74 \pm 0.18$ & $0.23 \pm 0.13$ \\
\hline AGS37 & $0.63 \pm 0.18$ & $1.10 \pm 0.16^{*}$ & $0.28 \pm 0.10$ \\
\hline AGS38 & $0.58 \pm 0.16$ & $1.00 \pm 0.16^{*}$ & $0.32 \pm 0.10$ \\
\hline AGS39 & $0.80 \pm 0.23 *$ & $0.98 \pm 0.28$ & $0.25 \pm 0.14$ \\
\hline
\end{tabular}

Notes. For each galaxy, the flux used (as explained in F18 and F20a) is indicated by an asterisk. The absence of a size indicates a nonconvergence of uvmodelfit. For AGS15, we obtained new 2 mm ALMA data for this galaxy, (project 2018.1.01079.S PI: M. Franco; see Zhou et al. 2020), which led us to revise our hypothesis that the source was compact and to favor the uvmodelfit flux which is in agreement with the $2 \mathrm{~mm}$ flux and the flux at $850 \mu \mathrm{m}$ (Cowie et al. 2018). 